Chapter 2

\title{
Electric Power Quality Recognition and Classification in Distribution Networks
}

\author{
Soliman Abdel-Hady Soliman and \\ Rashid Abdel-Kader Alammari \\ Additional information is available at the end of the chapter \\ http://dx.doi.org/10.5772/53081
}

\section{Introduction}

The power quality problem is now of a great concern to electric utilities of power industry and they are trying hard to supply their customers with a good quality of power especially in the open market. Due to the wide spread use of power electronics in every place in the power industry, the power supplied to the customers are now distorted in either the voltage signal or current signal or both of them. This distortion has a great effect on the sensitive equipments and may cause interruption to such equipments that result in very expensive consequence. It has been reported that $30 \%$ voltage sag for very short duration can reset programmable controllers for the entire assembly line. As such an accurate algorithm is needed for identification and measurements of these events

Power quality involves how close the voltage waveform is to being a perfect sinusoid with a constant frequency and amplitude. Historically, it has been the utilities' responsibility to provide a "clean" voltage waveform, and most customers' load did no affect the quality of their power. Today, a new factor, harmonics, has been added to the power quality scenario because utility customers, including residential ones, are using electronic devices that require non-sinusoidal currents, currents rich in harmonics.

The presence of power system harmonics is not a new problem; it has been well known since the first generator was built. However, nowadays due to the widespread use of electronic equipment, arcing devices, such as arc furnaces and equipment with saturated ferromagnetic cores, such as transformers, power engineers pay more attention to power system harmonics. The presence of voltage and current waveform distortion is generally expressed in terms of harmonic frequencies that are integral multiples of the power system nominal frequency. It is 
a steady phenomenon in a power system, and it is completely different from the distortion results from the transient, such as faults, in a power system.

The subject of harmonics has been deemed so important that there are international conferences dedicated to the subject, working groups and committees in international engineering societies, which deal only with harmonics, and several dedicated books on the topics. Power system, harmonics are become one of the important index to the power quality issues these days. Good power quality means less distortion, less harmonics, in the voltage and current sources.

Good harmonic prediction requires clear understanding of two different but closely related topics. One is the non-linear voltage/ current characteristic of some power system components and its related effect, the presence of harmonic sources. The main problem in this respect is the difficulty in specifying these sources accurately. The second topic is the derivation of the suitable harmonic models of the predominantly linear network components, and of the harmonic flows resulting from their interconnection. This task is made difficult by insufficient information on the composition of the system loads and their damping to harmonic frequencies. Further impediments to accurate prediction are the existence of many distributed nonlinearities, phase diversity, the varying nature of the load, etc.

To assess the quality of delivered power, especially in open markets, it is necessary to estimate the harmonic components in a power system. The quality of power delivered necessitates knowledge regarding the magnitude of harmonic components and phase angle of these components. The reduction of harmonics in a system means good system quality. Installing filters at feeding points along the network can do this if the harmonic components magnitudes as well as their phase angles are known in advance.

Over the past 25 years major improvements in the field of signal theory have been achieved and many algorithms and techniques have been published in the literature. In Ref. [1] an algorithm for tracking the voltage envelope based on calculating the energy operator of a sinusoidal waveform is presented. It is assumed that the frequency of the sinusoidal waveform is known and a lead-lag network with unity gain is used. An approach to power quality assessment based on real-time (RT) hardware-in-the-loop (HIL) simulation is proposed in Ref. [2]. The RT-HIL platform is being used for power quality studies of NAVY all-electric ships. Ref. [3] describes an analytical method for evaluating the voltage sag performance of a powersupply distribution network, different evaluation algorithms are proposed to account for each type of sensitivity.

Ref. [4] proposes a fuzzy pattern recognition system for power quality disturbances. It is a twostage system in which a multi-resolution S-transform is used to generate a set of optimal features vectors in the first stage. In the second stage a fuzzy logic-based pattern recognition system is used to classify the various disturbances waveforms generated due to power quality violations. The Teager energy operator (TEO) and the Hilbert transform (HT) are introduced in Ref. [5] as effective approaches for tracking the voltage flicker levels. It has been found that TEO and HT are capable of tracking the amplitude variations of the voltage flicker and supply frequency in industrial systems with an average error $3 \%$. Root-mean-square (rms) calculation 
is a popular method adopted in power system parameter classification such as voltage sag classification and relay protection. Ref. [6] studies the characteristics of the rms method, for both the single-frequency and mixed-frequency signals. Analysis is made on its dependence on sampling rate, sampling window size, as well as point-on-wave through strict mathematical deductions.

Ref. [7] presents a control technique for flicker mitigation. This technique is based on the instantaneous tracking of the measured voltage envelope. The ADALINE (ADAptive LINear) neuron algorithm and the Recursive Least Square (RLS) algorithm are introduced for the flicker envelope tracking. Presented in Ref. [8] is a fuzzy-expert system for automated detection and classification of power quality disturbances. The types of concerned disturbances include voltage sags, swells, interruption, switching transients, impulses, flickers, harmonics and notches from a signal that is available in sampled form. Fourier transform and wavelet analysis are utilized to obtain unique features for the waveforms. Ref. [9] and Ref. [10] present concepts based on fuzzy expert system for power quality study, the concept integrates the power system modeling, classifying and characterizing for power quality events, studying equipment sensitivity to the event disturbance, and locating point of event occurrence into one unified frame. Both Fourier and wavelet analyzes are applied for extracting distinct features of various types of events as well as for characterizing the events. Ref. [11] presents a technique for learning power- quality waveforms based adaptive neuro-fuzzy systems to learn power quality signature waveforms. Ref. [12] compared three signal processing tools for power quality analysis; the continues wavelet transform, the multi-resolution analysis and the quadratic transform. It has been concluded that the continues Wavelet transform is as reliable as these methods but has been the advantage of giving directly the magnitude of the $50 / 60 \mathrm{~Hz}$ signal, and is suitable for quantifying power quality when detecting and measuring voltage sags, transients over voltages or flicker. None of these techniques are as adequate to detect and measure the voltage magnitude of harmonic content as the Fourier transform. Reference [15] presents an optimal measurements scheme for tracking the harmonics in power system voltage and current waveforms. The proposed scheme was based on Kalman filtering. Reference [15] implements the well-known LES algorithm for identification and measurement of power system harmonics. The mathematical model for the identification and measurement process is presented in this reference. The samples used in this reference are for one of the three- phase voltage or current signals. Reference [16] presents a comparative study for power system harmonic estimation, where it compares the results obtained using discrete Fourier transform (DFT), the well known least errors square (LES) parameter estimation algorithm and the least absolute value (LAV) parameter estimation algorithm. It has been concluded that the three algorithms produce the same estimate, if the signal under study is free of noise. However, if some data samples are missed, the least absolute value produces better estimates than the DFT and LES algorithms. The algorithms in this reference use the samples of a one-phase voltage or current signal. An approach based on singular value decomposition (SVD) for estimating harmonic components in a power system is presented in Reference [17]. Three different techniques are investigated in this reference; the standard averaged SVD, the total LS and double SVD. Reference [18] implements the neural network in its analogue form for estimation of harmonics. It has been shown that such problem formulation leads to a quadratic objective 
of the global minimum, which can be found by using simple electronic circuitry in real time. Reference [19] presents an approach for the estimation of harmonic components of a power system using a linear adaptive neuron called Adaline. The proposed estimator tracks Fourier coefficients of signal data corrupted with noise and decaying DC components. Reference [28] develops a fast Newton type solution of the six-pulse rectifier and dc system in the harmonic domain. The nonlinear equations are solved using Newton's method, which employs a Jacobian matrix of partial derivatives. A twelve states Kalman filtering algorithm is applied in Reference [29], using an 8-bit microprocessor, for continues real-time tracking of the harmonics in the voltage or current waveforms of a power system to obtain in real time the instantaneous values for a maximum of six harmonics as well as the existing harmonic distortion. Reference [30] reviews the problems associated with direct application of the Fast Fourier Transform to compute harmonic levels of non-steady state distorted waveforms, and various ways to describe recorded data in statistical terms. Reference [31] presents an approach based on fuzzy linear regression for the measurement of power system harmonic components. The nonsinusoidal voltage or current waveform is written as a linear function. The parameters of this function are assumed to be fuzzy numbers having certain middle and spread value. The problem in this reference is formulated as a linear optimization problem, where the objective is to minimize the spread of voltage or current samples. The on-line digital measurement on power systems for the power quality analysis under non-sinusoidal conditions is considered in Reference [32]. The proposed instrument, in this reference, adopts a floating point digital signal processing (DSP) hosted on a IBM PC and interfaced with a special high-speed data acquisition system (DAS). Voltage and current waveforms are acquired and processed by using a fast recursive least-square (FRLS) measurement algorithm. Using such an algorithm, different quantities are obtained, such as the current and voltage rms values, their harmonic content, the active power, the harmonic active power, the power factor, etc. Reference [33] applies a technique to the computation of individual harmonics in digital protections, where only certain isolated harmonics, rather than the full spectrum, are needed. This leads to $\mathrm{O}$ $\left(\log _{2} \mathrm{~N}\right)$ computations per harmonics. A technique based on modeling and identification method is proposed in Reference [34] using a mathematical model describing the signal in question. The recursive least-square-error identification algorithm is used to identify the harmonic parameters. These are including the frequency, the amplitude and phase angle. Because of the limitations associated with conventional algorithms, particularly under supplyfrequency drift and transient situations, an approach based on non-linear least squares parameter estimation has been proposed in Reference [35]. To reduce the computational time the Hopfield type feedback neural networks for real-time harmonic evaluation. The neural network implementation determines simultaneously the supply frequency variation, the fundamental-amplitude/phase variation as well as the harmonics-amplitude variation. Reference [36] considers state estimation of harmonic signals with time varying magnitudes. Harmonic signals are modeled using elliptical set-theoretic methods and an optimal reducedorder estimator, which has one-half the dimension of the state vector, is developed for predicting the unknown time-varying harmonic magnitudes. Reference [37] proposes the optimization of spectrum analysis to reduce the restriction on Fast Fourier Transform (FFT) [38] resulting mismatch the frequency scale with signal characteristics. By using this method 
both of the picket-fence effect and the leakage effect are reduced, and it makes the harmonic parameters show on spectrum more accurately. Reference [39] proposes a harmonic model based on Wavelet Transform (WT) for on-line tracking of power system using Kalman filtering. The close relation between the Wavelet and Multi-resolution analysis is utilized to express the harmonic magnitudes and phase angle as a sum of Wavelet and scaling function.voltage sags define as an rms reduction in the AC voltage, at the power frequency, for duration from a half a cycle to a few second. If the voltage drops below normal level for several cycles it would affect the critical load and cause shutdown to these loads. Voltage sags constitute the majority of power line problem representing about $60 \%$ of all problems. In addition to these quantities, sags also characterize by unbalance, non-sinusoidal wave-shapes and phase angle shift (phase jump). These factors are important for determining the behavior of ac motor drives during sags [40]. The main sources for the voltage sags are start-ups of large motors, sudden increase in line loads, electrical faults on utility power lines caused by animals, trees, storms, or other objects in contacts with power lines. A majority of faults on a utility system are single-line-toground faults (L-G). During L-G faults, the voltage on the faulted phase goes to nearly zero volts at the fault location. The corresponding voltage at a customer bus depends on the system configuration, location of the fault, the impedance of the system upstream of the fault, the feeder impedance, the distance of the fault and the transformer connections between the faulted system and customer bus. Furthermore, Electronic loads that pull large currents such as copy machines, laser printers can cause voltage sags. Further more, loose wiring in the distribution installation can cause voltage sags [14]. To quantify the effect of sensitive equipment to voltage sags, it is necessary to characterize the parameters of voltage sags. Most often, voltage sags characterize by a duration and depth parameter and represent in a two-dimensional rms voltage magnitude versus duration plot. This simplified representation of voltage sag characteristics does not take into account the different in individual phase voltages (voltage asymmetry or unbalance) and the associated phase angle shift during voltage sag. Furthermore, it does not take into account the non-sinusoidal nature of the voltage waveform during the sag. The magnitude of voltage sag can be determined from the rms voltage. As long as the voltage is sinusoidal, it does not matter whether rms voltage, fundamental voltage, or peak voltage used to obtain the sag magnitude. But especially during a voltage sag this is often not the case.[15] Chapter 4 in Ref. [15] explains in details the voltage sag characterization, the different available techniques used to measure the voltage sags at different modes of operation of power systems Fast Fourier Transform used to measure the phase angle jumps using the moving window length technique.

In Reference [42], a method for voltage disturbances such as voltage sags, voltage swells, flicker, frequency change in the utility voltage, and harmonic distortion of a single-phase or poly phase voltage disturbances is explored The algorithm is based on the theory that allows a set of three-phase voltages be represented as dc voltages in a $d-q$ synchronous rotating frame. In this case, the utility input voltages are sensed and then converted to dc quantities in the $d-$ $q$ reference frame. The output of the algorithm is compared with a set of reference voltages, and the error is used as a measure to such disturbances. Reference [43] presents a Monte Carlo based approach to evaluate the maximum voltage sag magnitudes as well as the voltage unbalance in transmission systems. In this context, investigations have been conducted on a 
system model taking into consideration the uncertainty in several factors associated with the practical operation of a power system.

Reference [44] has discussed the limitations of the conventional sag characterizing method and proposed a new sag characterizing method. The conventional method overestimates the nonrectangular sag and cannot reflect the exact effect of voltage sag according to the voltage tolerance characteristics. The method has approximated the voltage profile during voltage sag using order radical root function. It has modified the voltage sag duration using the known parameters, and which are measured at PQ monitors. With the modified sag duration, the method has evaluated the effect of voltage sag correctly. Moreover, it can be applied to not only rectangular sag but also nonrectangular sag.

Reference [45] proposes the concept of "voltage sag state estimation" and associated algorithms to achieve this goal. The method has the characteristics: 1) It makes use of the radial connection characteristic of a distribution feeder, 2) it is based on a limited number of metering points, and 3) it employs a least-square method to predict the sag profile along a distribution line. The results of sag state estimator can be used to calculate the feeder power quality performance indices such as the System Average RMS Frequency Index (SARFIx). Reference [46] presents a technique for accurate discrimination between transient voltage stability and voltage sag by combining damped sinusoids-based transient modeling with neural networks. Transient modeling is accomplished by energy adapted matching pursuits with an overcomplete dictionary of damped sinusoids. In this approach, the information provided by the damped sinusoids-based transient modeling stage is applied to a Neural Network, which determine in a fast and accurate fashion the class to which the waveform belongs. In Reference [47] a power quality assessment method was proposed. Test results show that the method can tolerate highly distorted voltages, significant sudden frequency change, and three-phase voltage sags, but it cannot tolerate certain short-term phase-shifted single-phase voltage sags. In Reference [40] a control algorithm for the (Distributed Generation) DG interface based on the Hilbert transform (HT) is presented. The HT is employed as an effective technique for tracking the voltage flicker levels in distribution systems. The technique can be used on-line tracking of voltage flicker. The accurate tracking of the HT facilitates its implementation for the control of flicker mitigation devices. The HT realized with long filter length provides a minimum error in tracking the voltage envelope but with higher computation cost and a larger delay time than that of the short filter length. The HT can surpass the Kalman Filter in voltage tracking in a sense that it requires less computation effort and avoid the pitfalls of the FFT. In Reference [48], an algorithm for tracking the voltage envelope based on calculating the energy operator of a sinusoidal waveform is presented. This algorithm is used to evaluate the instantaneous changes in the amplitude and so track the envelope of the waveform. The algorithm is fast and robust and uses only a few samples to calculate the energy. It is not sensitive to the noise or the distortion in the waveform. The results show the capability of the algorithm to track different shapes of envelopes associated with high signal-to-noise ratio (SNR). However, there will be a delay between the actual and the tracked envelope, due to using the lead/lag networks 
A prototype wavelet-based neural network classifier for recognizing power-quality disturbances is implemented and tested in Reference [50], under various transient events. The discrete wavelet transform (DWT) technique is integrated with the probabilistic neuralnetwork (PNN) model to construct the classifier. First, the multi resolution-analysis technique of DWT and the Parseval's theorem are employed to extract the energy distribution features of the distorted signal at different resolution levels. Then, the PNN classifies these extracted features to identify the disturbance type according to the transient duration and the energy features. Various transient events are tested, such as, momentary interruption, capacitor switching, voltage sag/swell, harmonic distortion, and flicker. The results show that the classifier can detect and classify different power disturbance types efficiently. Reference [51] proposes a wavelet-based extended fuzzy reasoning approach to power-quality disturbance recognition and identification. To extract power-quality disturbance features, the energy distribution of the wavelet part at each decomposition level is introduced and its calculation mathematically established. Based on these features, rule bases are generated for extended fuzzy reasoning. The power-quality disturbance features are finally mapped into a real number, in terms of which different power-quality disturbance waveforms are classified. One prominent advantage of this approach is that the power-quality engineers' knowledge about features of the disturbance waveforms can be easily included into the algorithm using linguistic description.

Reference [52] presents a procedure for stochastic prediction of voltage sags based on the Monte Carlo method. A medium size distribution network is used to analyze, the convergence of the Monte Carlo method, the influence of protective devices and the importance of voltage sag indices is studied. This reference has presented the scope and advantages of an EMTPbased procedure for voltage sag analysis. The aim of a stochastic prediction is not only to deduce the number of voltage sags but also the number of trips of sensitive equipment. Therefore, the representation of equipment sensitivity is also required. In Reference [53] the Recursive Least Square (RLS) algorithm are introduced for the flicker envelope tracking. Both the ADALINE and the RLS algorithms are used to track the voltage envelope. The difference between the estimated envelope and the required voltage level is passed to the controller to obtain the required reactive power to compensate flickers. A fast response, accurate tracking, and robustness of the proposed control system are revealed from the results. In addition, the performance of the ADALINE and the RLS algorithms for the estimation of flicker envelopes are investigated.

Reference [54] presents and verifies a voltage sag detection technique for use in conjunction with the main control system of a DVR. A problem arises when fast evaluation of the sag depth and phase shift is required, as this information is normally embedded within the core of a main DVR control scheme and is not readily available to either user monitoring the state of the grid or parallel controllers. The voltage sag detection method in this reference proposes a matrix method, which is able to compute the phase shift and voltage reduction of the supply voltage much quicker than the Fourier transform or a PLL. DPQ sites have widely dispersed power quality. Many sites have many more sags than other sites. Rural sites have many more sags and momentary interruptions than suburban and urban sites. The three strongest indicators 
of voltage sags are 1) circuit exposure, 2) lightning, and 3) a term with transformer size and number of feeders. A linear model can predict sags based on a small number of site characteristics. Load density and three-phase circuit exposure most strongly affect momentary [55]. In Reference [56] a potential problem area in using RMS values in power quality assessment are identified and discussed. The RMS can be computed either using a fixed window (s-RMS) or a moving average technique (m-RMS). In both cases, RMS is a function of window length, and is a constant function for periodic signals of fundamental period.

Reference [57] presents an expert system for automatic classification of power quality recordings. The classification procedure is based on segmenting the voltage waveforms in points of sudden changes in the fundamental magnitude. Based on the segmentation results, a set of classification modules is utilized to classify the event. Classification is based on features extracted from the voltage waveforms. The system successfully classifies the largest part of the recordings. The only problems that are found are related with either the failure in detecting very small changes in the voltage magnitude or the time resolution problems of the magnitude estimation and the detection. The expert system enables fast and accurate analysis of large databases and classification of the recordings in terms of the origin. Event classification (instead of disturbance classification) offers the means for better understanding and description of the operation of the system in terms of power quality.

Reference [58] uses the continuous wavelet transform to detect and analyze voltage sags and transients. Recursive algorithm is used and improved to compute the time-frequency plane of electrical disturbances. Characteristics of investigated signals are measured on a timefrequency plane. A comparison between measured characteristics and benchmark values detects the presence of disturbances in analyzed signals and characterizes the type of disturbances. Duration and magnitude of voltage sags are measured; transients are located in the width of the signal. Furthermore, meaningful time and frequency components of transients are measured. Detection and measurement results are compared using classical methods. This algorithm enables very accurate time location and magnitude measurements of voltage sags and meaningful transient identifications. Furthermore, the method enables an accurate classification of transient events to be performed, and characteristics are easily read from the time-frequency plane. Reference [59] shows that it is possible to use sampled voltage and current waveforms to determine on which side of a recording device a disturbance originates. This is accomplished by examining the energy flow and peak instantaneous power for both capacitor energizing and voltage sag disturbances. By examining sampled voltage and current waveforms, it is possible to make a judgment as to which side of a recording device a power quality disturbance event originates. This is accomplished by examining the disturbance power and energy flow and the polarity of the initial peak of the disturbance power. If several recording devices are available in a network, the source of the disturbance may be pinpointed with a higher degree of accuracy.

2. $\alpha \beta$-Transformation for identifying the power quality events (Clarkes transformation) $\alpha \beta$ Transformation (Clarks Transformation) is a dc transformation used to transfer the threephase ac system of voltage or current to a dc system. This section presents the application of this transformation to identifying the power quality events, such as harmonics, voltage 
sages, flicker, swell and transients. This transformation can be implemented for a single phase or three- phase system of voltage. The phasor voltage resulting from the combination of $V_{\alpha}$ and $V_{\beta}$ has a magnitude proportional to the system operating voltage and rotates with a speed of the frequency of the system voltage. The estimated shape of the phasor voltage over the data window size gives the nature of the power quality event. The proposed technique can be implemented in off-line or on-line modes. Simulated results, for different types of events are presented in the text and the results show excellent identification using the proposed technique.

\subsection{Modeling the voltage signals [1]}

The three phase voltages of the power systems can be written at any sample $k, k=1, m, m$ is the total number of samples available

$$
\begin{aligned}
& \mathrm{v}_{\mathrm{a}}(k)=V_{m a} \sin \left(\omega k \Delta T+\varphi_{a}\right)+\zeta_{a}(k) \\
& \mathrm{v}_{\mathrm{b}}(k)=V_{m b} \sin \left(\omega k \Delta T+\varphi_{b}-120^{0}\right)+\zeta_{b}(k) \\
& \mathrm{v}_{\mathrm{c}}(k)=V_{m c} \sin \left(\omega k \Delta T+\varphi_{c}+120^{0}\right)+\zeta_{c}(k)
\end{aligned}
$$

Where we define the parameters in (1) as:

$V_{m}$ is the signal amplitude

$\omega$ is the signal nominal frequency

$\Delta T$ is the sampling time $=\frac{1}{F_{s}}, F_{s}$ is the sampling frequency

$k$ is the sampling step; $k=1, . ., m$

$\varphi$ is the signal phase angle

$\zeta_{a}(k), \zeta_{b}(k)$ ,$\zeta_{c}(k)$

are the noise terms which may contain harmonics

The well-known $\alpha \beta$ - transformation for the three-phase signal is given as:

$$
\left[\begin{array}{c}
v_{\alpha}^{\alpha}(k) \\
v_{\beta}(k)
\end{array}\right]=\sqrt{\frac{2}{3}}\left[\begin{array}{ccc}
1 & -0.5 & -0.5 \\
0 & \frac{\sqrt{3}}{2} & \frac{-\sqrt{3}}{2}
\end{array}\right]\left[\begin{array}{c}
v_{a}(k) \\
v_{b}(k) \\
v_{c}(k)
\end{array}\right]
$$

Equation (2) can be rewritten as: 


$$
\begin{aligned}
& v_{\alpha}(k)=\sqrt{\frac{2}{3}}\left[v_{a}(k)-0.5 v_{b}(k)-0.5 v_{c}(k]\right. \\
& \mathrm{v}_{\beta}(\mathrm{k})=\sqrt{\frac{2}{3}}\left[\frac{\sqrt{3}}{2} \mathrm{v}_{\mathrm{b}}(\mathrm{k})-\frac{\sqrt{3}}{2} \mathrm{v}_{\mathrm{c}}(\mathrm{k})\right]
\end{aligned}
$$

For $\mathrm{m}$ samples of three-phase signals, $\mathrm{m}$ samples for $v_{\alpha}(k), v_{\beta}(k)$ are obtained. By using this transformation, harmonics of order three and their multiples are suppressed. Equation (3) and (4) gives the transformed voltage $v_{\alpha}(k), v_{\beta}(k)$ at any sample $k$. The complex voltage phasor formed from these two voltages which having the same frequency $\omega$ and phase angle $\varphi$ as the original three phases is:

$$
\begin{aligned}
v(k) & =v \alpha^{(k)+j v} \beta^{(k)} \\
& =V(k) e^{j(\omega k \Delta T+\varphi)}
\end{aligned}
$$

Where the amplitude $V(k)$ of the complex signal is calculated at any sample $k$ as:

$$
V(k)=\left[v_{\alpha}^{2}(k)+v_{\beta}^{2}(k)\right]^{\frac{1}{2}}
$$

and the rms magnitude is given as:

$$
V=\frac{1}{m} \sum_{k=1}^{m}\left[v_{\alpha}^{2}(k)+v_{\beta}^{2}(k)\right]^{\frac{1}{2}}
$$

Equation (6) gives the value of phasor voltage at any instant $k$, while equation (7) is used to calculate the rms value of the phasor voltage.

The phase angle of this transformed voltage is:

$$
\theta(k)=\tan ^{-1} \frac{v_{\beta}(k)}{v_{\alpha}(k)}
$$




\subsection{Testing the algorithm}

The proposed algorithm explained in the previous section is used to identifying the power quality events for a three phase balanced voltage. These are including voltage swell, voltage flicker, momentary interruption, voltage sags, and harmonics and voltage transients due to switching on/off capacitors used in reactive power compensation.

\subsubsection{Voltage swell}

In this test a voltage swell is assumed on the system, over voltage, for a short period. The rms value for the complex phasor is calculated, using equation (7) as well as the phasor phase angle in radian is computed using equation (8)

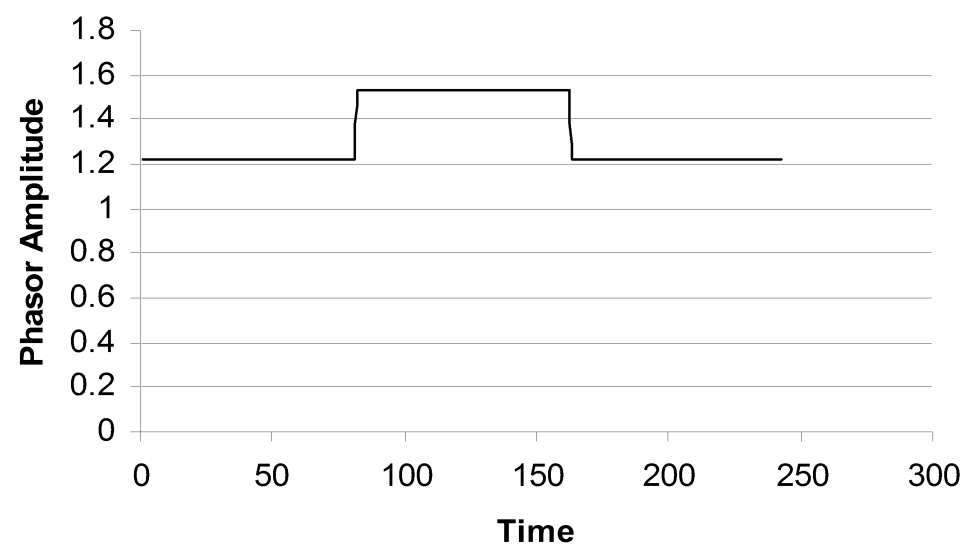

Figure 1. A system of three-phase voltage experiences a voltage swell.

Figure 1 gives the amplitude, rms value and the phase angle of the phasor for a system of threephase voltage experiences a voltage swell. It can easily be noticed, from the phasor amplitude that the voltage is experienced a voltage swell of magnitude 1.53 , normal voltage is $1.224 \mathrm{p} . \mathrm{u}$, during the time that equivalent to sample number 83 to the time that equivalent to sample number 163.

\subsubsection{Voltage flickers}

In this test we assume that the three phase voltages are experienced a flicker the complex phasor of the voltage is extracted from the available samples for the three phase voltages.

Figure 2 gives the results obtained. Examining this figure reveals that this amplitude is modulated amplitude during the data window size under investigation, i.e. looking to this figure one can conclude that a flicker signal is imposed on the nominal voltage signal. 


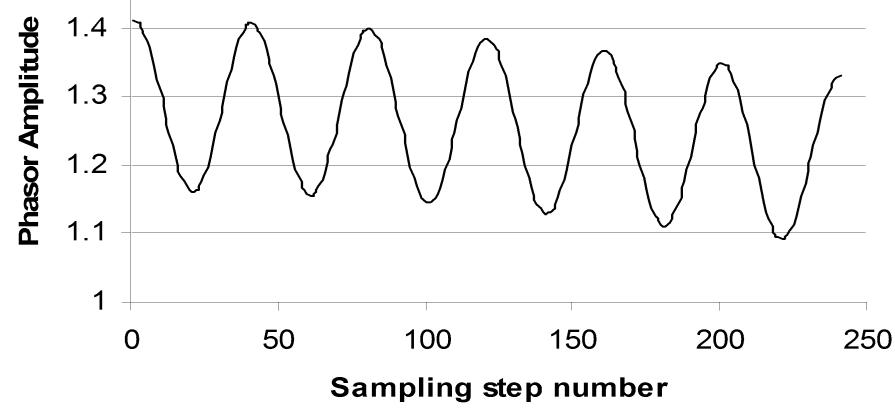

Figure 2. Phasor amplitude for a voltage flicker

\subsubsection{Momentary interruption}

A momentary interruption for a small period of time is implemented in this test, and then the voltage is restored again. Figure 3 gives the phasor amplitude when the system voltage experienced a momentary interruption. Examining this curve reveals that the proposed algorithm is succeeded in estimating the voltage amplitude and the period of interruption.

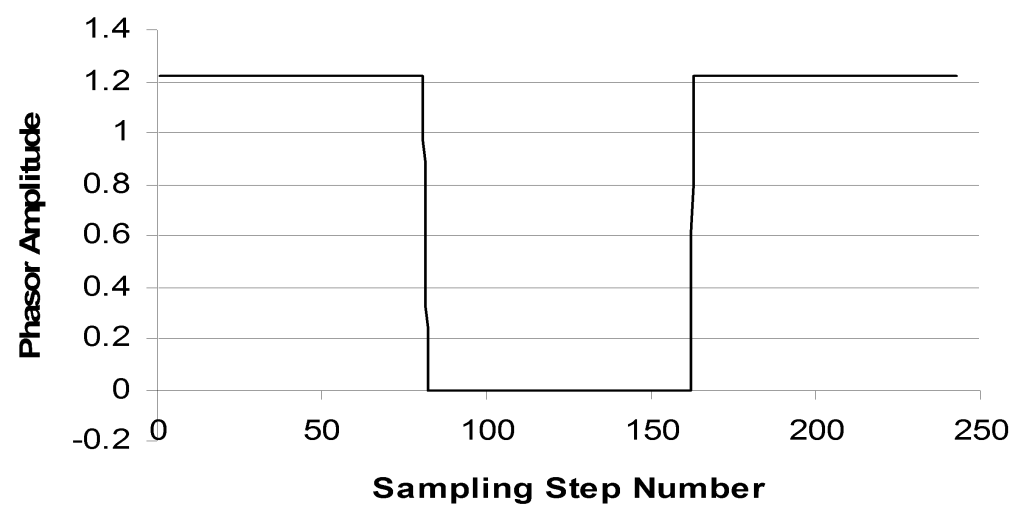

Figure 3. Phasor Amplitude for a momentary interruption

This figure also reveals that the phasor amplitude tends to zero from the time equivalent to the samples between 85 and 162, the time of interruption is (162-85)* sampling time, and after the sample number 162 the system voltage is restored again to nominal voltage.

\subsubsection{Voltage sags}

In this test voltage is assumed to experience voltage sag, where the voltage is reduced to 0.6 $\mathrm{pu}$, the voltage phasor amplitude of equation 6 is calculated. Figure 4 gives the results obtained, 
examining this curve reveals that the proposed algorithm is succeeded in estimating the voltage amplitude as well as the sags period.

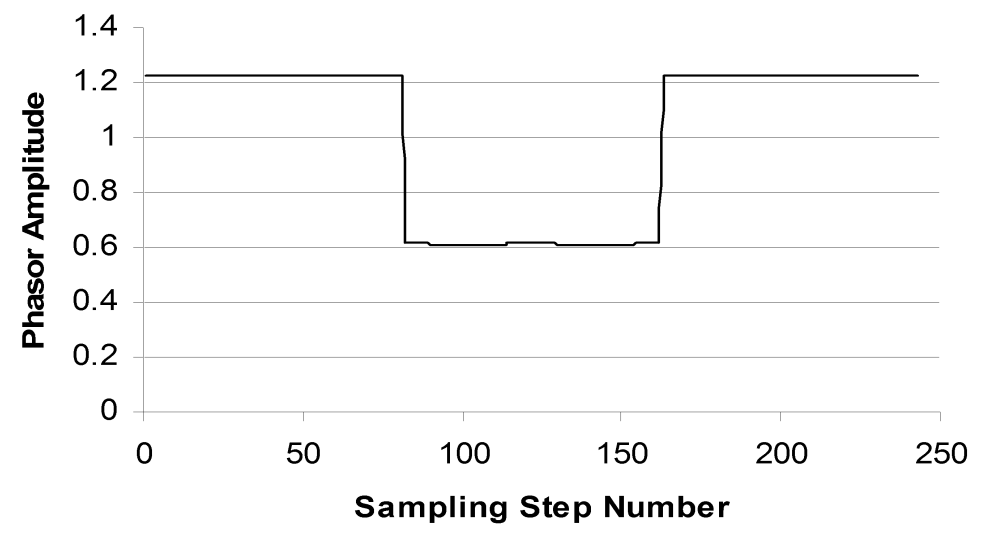

Figure 4. Phasor amplitude for a Voltage experiences voltage sag

\subsubsection{Voltage transients}

In this test the three-phase voltage signal is experienced voltage transient, this may come from faults and/or lightning. The phasor amplitude is calculated using the proposed algorithm.

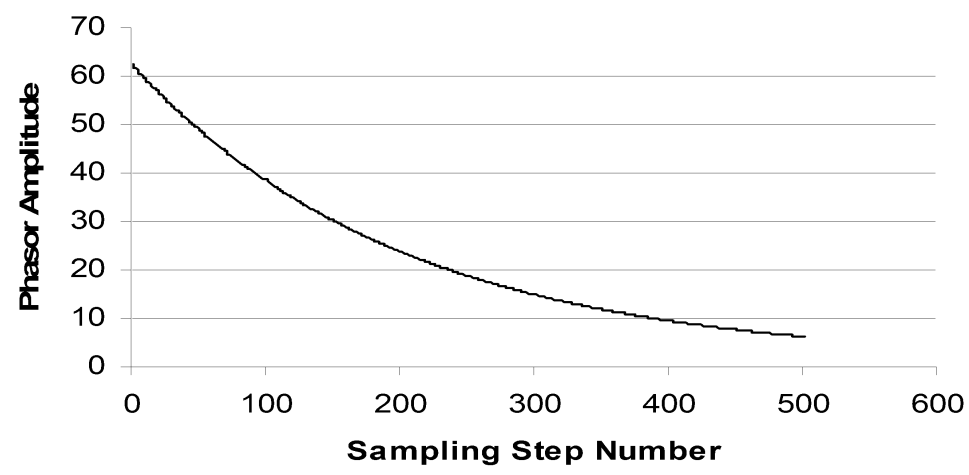

Figure 5. Phasor amplitude for voltage experiences voltage transients

Figure 5 depicts the results obtained. Examining this curve reveals that the proposed transformation is succeeded in estimating the voltage amplitude as well as the transient period. 


\subsubsection{Harmonics}

The voltage signal in this test is assumed to be polluted by harmonics up to the fifth order, and the proposed algorithm is used to estimate the voltage phasor amplitude. Figure 6 depicts the results obtained. If we examine this figure we can conclude that the phasor amplitude is a harmonically polluted one, and is following exactly the system voltage.

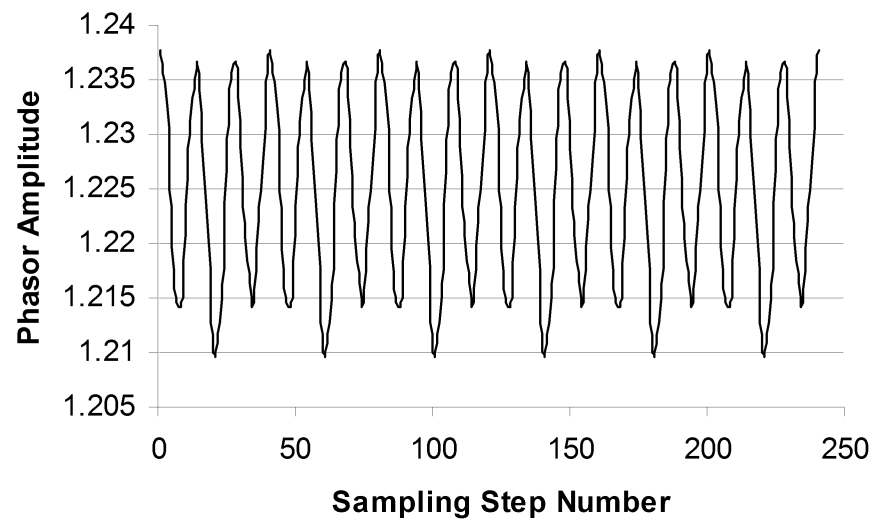

Figure 6. Voltage signal polluted with harmonics

\subsection{Conclusion}

We present, in this section, the application to $\alpha \beta$-Transformation for identifying and discriminating between different types of power quality events. Simulated examples for these events are presented in the body of the text and more results for the nature of the transformed voltages are given. It has been shown, through extensive runs, that if one of the phase is experienced a power quality event alone while the others are not, the algorithm is succeeded in identifying this events which is not the case of the other earlier algorithms in the past.

\section{Kalman filtering algorithm}

Power quality involves how close the voltage waveform is to being a perfect sinusoid with a constant frequency and amplitude. In this section, we review the applications of linear Kalman filtering algorithm for on-line electric power quality analysis. These applications include the measurement of harmonics and voltage sags. Mathematical models for each problem are developed to suite the filter and tested using simulated examples.

\subsection{Harmonics model}

The voltage signal can be written as Fourier series as: 


$$
v(t)=\sum_{n=0}^{N} \sqrt{2} V_{n} \cos \left(n \omega t+\phi_{n}\right)
$$

A d.c component may exist in the voltage signal, this occurs at $n=0$. Equation ( 9 ) can be written, for a specific number of harmonics, as:

Equation (9) can be rewritten as:

$$
\begin{aligned}
& v(t)=V_{o}+\sqrt{2} V_{1} \cos \phi_{1} \cos \omega t-\sqrt{2} V_{1} \sin \phi_{1} \sin \omega t+\sqrt{2} V_{2} \cos \phi_{2} \cos 2 \omega t-\sqrt{2} V_{2} \sin \phi_{2} \sin 2 \omega t \\
& +\sqrt{2} V_{3} \cos \phi_{3} \cos 3 \omega t-\sqrt{2} V_{1} \sin \phi_{3} \sin 3 \omega t
\end{aligned}
$$

In equation (10) we assume that the voltage signal is contaminated up to the third harmonic. But the incoming steps are generally enough to apply for any number of harmonics.

Define the following states as :

$$
\begin{aligned}
& X_{o}=V_{o} \\
& X_{1}=\sqrt{2} V_{1} \cos \phi_{1} \\
& Y_{2}=\sqrt{2} V_{1} \sin \phi_{1} \\
& X_{2}=\sqrt{2} V_{2} \cos \phi_{2} \\
& Y_{2}=\sqrt{2} V_{2} \sin \phi_{2} \\
& X_{3}=\sqrt{2} V_{3} \cos \phi_{3} \\
& Y_{3}=\sqrt{2} V_{3} \sin \phi_{3}
\end{aligned}
$$

Moreover, define the following coefficients as:

$h_{o}=1$

$h_{1}(k \Delta t)=\cos 2 \pi f k \Delta t$

$h_{2}(k \Delta t)=-\sin 2 \pi f k \Delta t$

$h_{3}(k \Delta t)=\cos 4 \pi f k \Delta t$

$h_{4}(k \Delta t)=-\sin 4 \pi f k \Delta t$

$h_{5}(k \Delta t)=\cos 6 \pi f k \Delta t$

$h_{6}(k \Delta t)=-\sin 6 \pi f k \Delta t$

Equation (10) can be written as: 


$$
v(k \Delta t)=X_{o}+X_{1} h_{1}(k \Delta t)+Y_{1} h_{2}(k \Delta t)+X_{2} h_{3}(k \Delta t)+Y_{2} h_{4}(k \Delta t)+X_{3} h_{5}(k \Delta t)+Y_{3} h_{6}(k \Delta t)
$$

In equation (12) $k=1,2, . ., m$, and $\Delta t$ is the sampling time.

Equation (12) can be written as an observation equation as:

$$
\left[\begin{array}{c}
v(\Delta t) \\
v(2 \Delta t) \\
\cdot \\
\cdot \\
\cdot \\
v(m \Delta t)
\end{array}\right]=\left[\begin{array}{ccccccc}
1 & h_{1}(k \Delta t) & h_{2}(k \Delta t) & h_{3}(k \Delta t) & h_{4}(k \Delta t) & h_{5}(k \Delta t) & h_{6}(k \Delta t) \\
1 & h_{1}(2 k \Delta t) & h_{2}(2 k \Delta t) & h_{3}(2 k \Delta t) & h_{4}(2 k \Delta t) & h_{5}(2 k \Delta t) & h_{6}(2 k \Delta t) \\
& & & & & & \cdot \\
\cdot & \cdot & \cdot & \cdot & \cdot & \cdot & \cdot \\
1 & h_{1}(m \Delta t) & h_{2}(m \Delta t) & h_{3}(m \Delta t) & h_{4}(m \Delta t) & h_{5}(m \Delta t) & h_{6}(m \Delta t)
\end{array}\right]\left[\begin{array}{c}
X_{0} \\
X_{1} \\
Y_{1} \\
X_{2} \\
Y_{2} \\
X_{3} \\
Y_{3}
\end{array}\right]
$$

Equation (13) can be written in vector form as:

$$
\underline{Z}=H \underline{\theta}+\underline{\xi}
$$

Where $Z$ is an $m \times 1$ samples of voltage, $H$ is an $m \times 7$ measurement matrix which can be calculated off line, $\theta$ is $7 \times 1$ parameters vector to be identified and $\xi$ is $m \times 1$ errors vector to be minimized. Note that the order of $H$ matrix as well as the vector $X$ depends on the number of harmonic expected to contaminate the voltage signal.

We assume that the system state equation is given by

$$
\underline{\theta}(k+1)=\phi \underline{\theta}(k)+\underline{v}(k)
$$

Where $\varphi$ is $7 \times 7$ state transition matrix, and it is assumed to be identity matrix. Moreover, $v(k)$ is $7 \times 1$ errors vector associated with the states when they move from stage $k$ to $k+1$. Now equations (14) and (15) are suitable for Kalman filter application 


\subsection{Computer experiments}

\subsubsection{Harmonics identification}

The voltage signal in this test is assumed to be contaminated with harmonics, $3^{\text {rd }}, 5^{\text {th }}$, and $7^{\text {th }}$ order. The voltage signal in this case is given as:

$v(t)=\sqrt{2}\left[\cos \left(\omega t+60^{\circ}\right)+0.25 \cos \left(3 \omega t+15^{\circ}\right)+0.15 \cos \left(5 \omega t-10^{\circ}\right)+0.05 \cos (7 \omega t)\right]$

Where $\omega=2 \pi f, f=50 \mathrm{~Hz}$, and it is assumed to be constant during the estimation process. This signal is sampled at frequency of $2000 \mathrm{~Hz}, 40$ samples per cycle.

Figure 7 shows the estimated fundamental, $3^{\text {rd }}, 5^{\text {th }}$ components when the voltage signal is modeled using up to the $7^{\text {th }}$ harmonic. While Figure 8 shows the estimated phase angle for each harmonics component including the fundamental component. The following observation can be concluded from these two Figures:

- Kalman filtering algorithm is succeeded in estimating the magnitude of harmonics components, as well as the phase angle of each component, providing that the voltage signal is accurately modeled

- It has been shown through extensive runs that the sampling frequency and number of samples do not affect the estimates of the magnitudes and phase angles of each harmonics component. Providing that the sampling frequency satisfies the sampling theory.

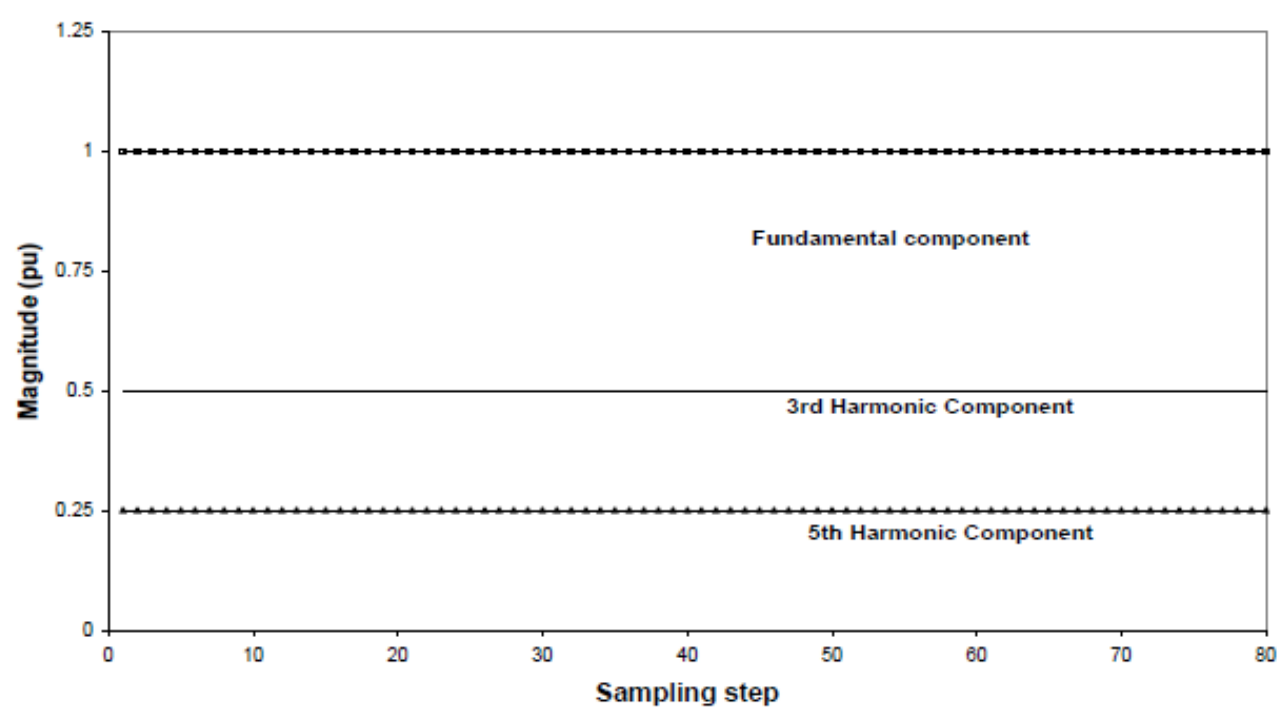

Figure 7. Estimated Magnitudes 


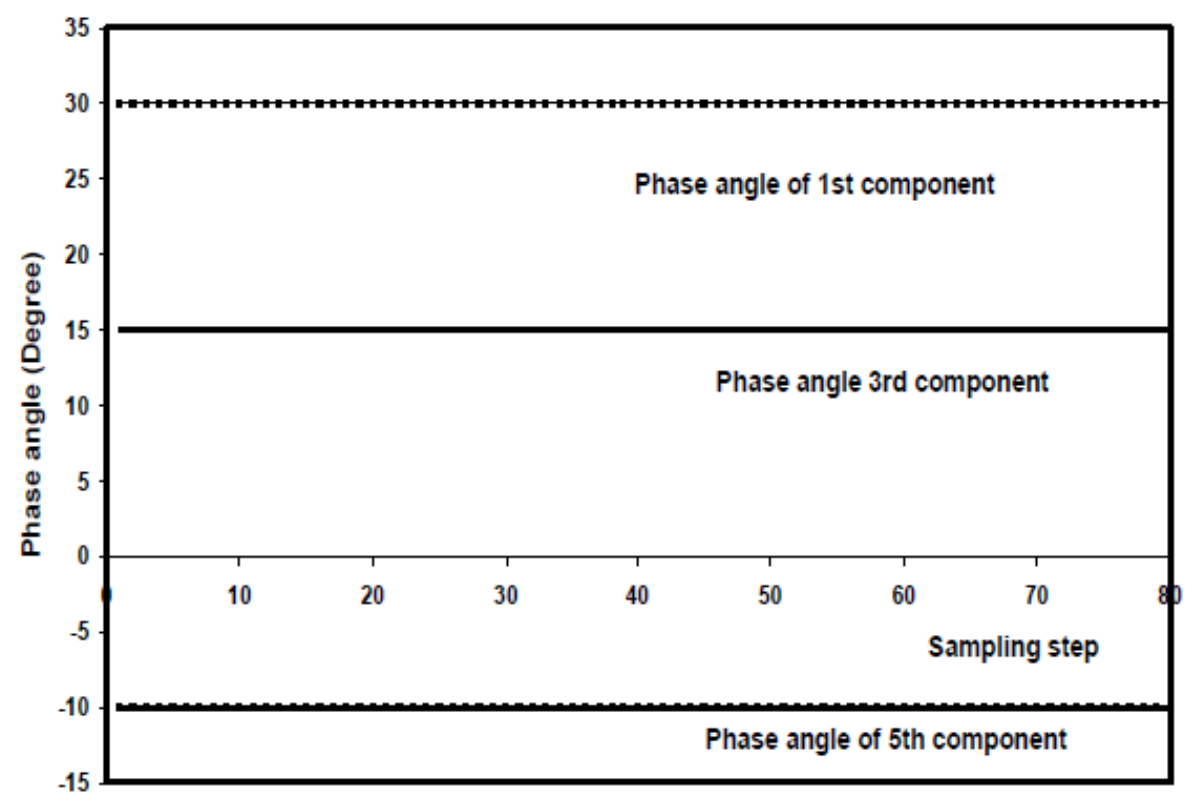

Figure 8. Estimated phase angle

Another test is conducted, where we modeled the voltage signal in the observation equation to take only the fundamental component i.e we assume that the voltage signal does not contaminated with harmonics, but in reality, it does. Figure 9 and 10 gives the estimated fundamental magnitude and its phase angle respectively.

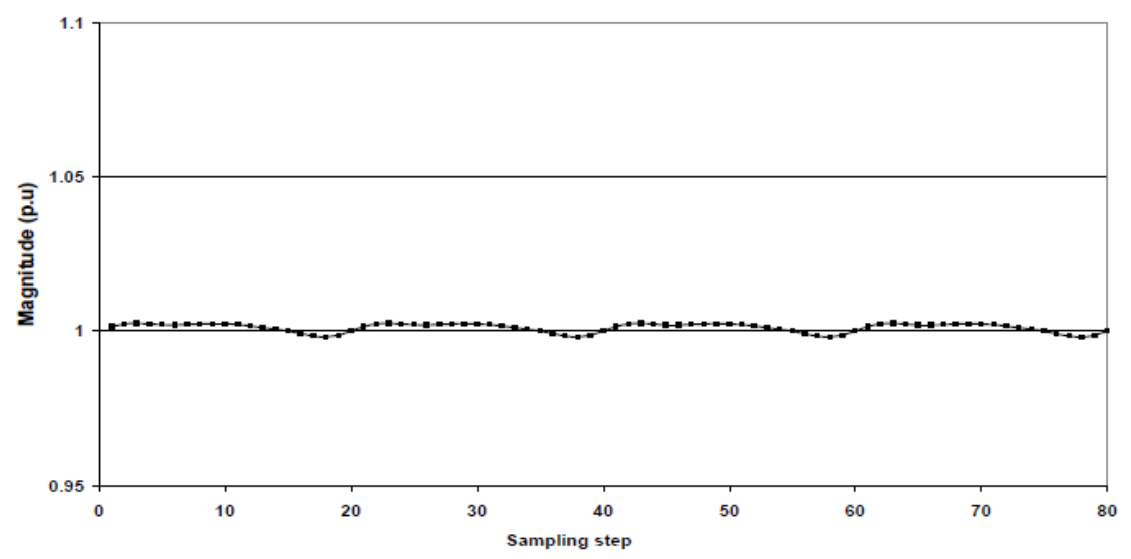

Figure 9. Estimated Fundamental Magnitude 


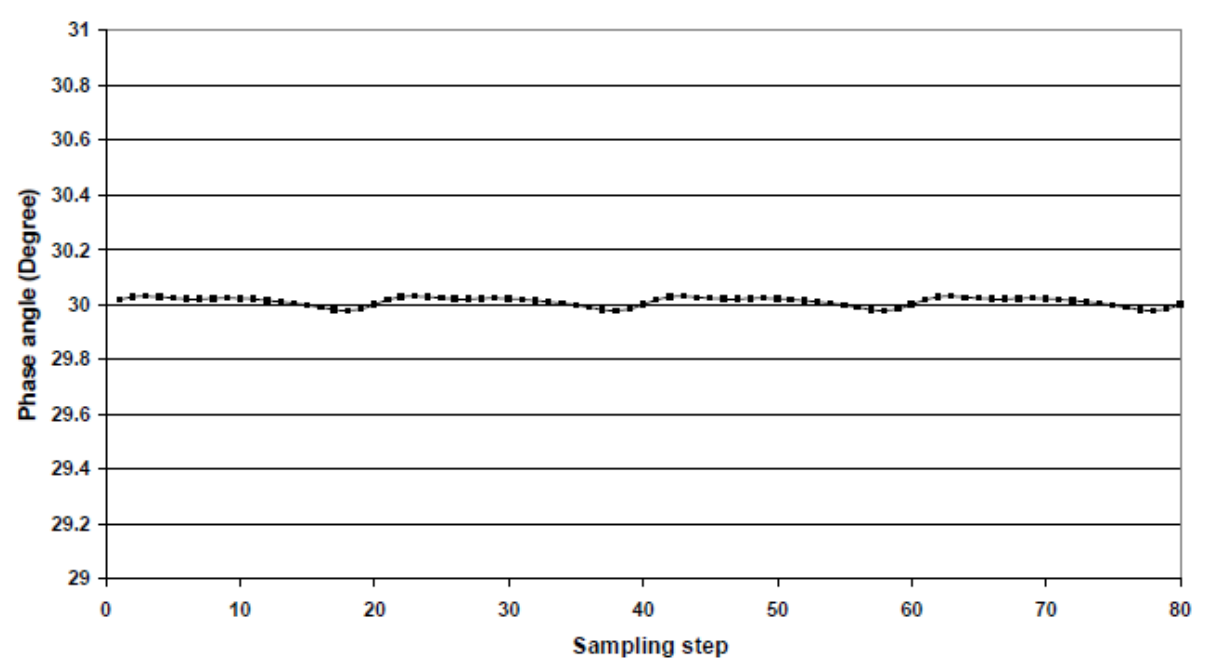

Figure 10. The estimated fundamental phase angle

Examining these curves reveals the following remarks:

- Although the fundamental component is the only one modeled in the waveform, good estimates are obtained for both amplitude and phase angle.

- The proposed algorithm can easily estimate the fundamental component and its phase angle using 20 samples, since the estimates are periodical, i.e a half cycle data window size is enough.

\subsubsection{Voltage sags}

\subsubsection{A pure sinusoidal voltage signal}

The source voltage in this case takes the following waveform equation:

$$
v(t)=\sqrt{2} V_{s} \cos \left(\omega_{0} t+60^{\circ}\right)
$$

Where $\omega_{\mathrm{o}}$ is the nominal frequency $=2 \pi \mathrm{f}_{\mathrm{o}}, \mathrm{f}_{\mathrm{o}}=50 \mathrm{~Hz}, V_{s}$ is the value of the voltage during the period of measurements, presage value, during sage and post sage voltage. In this simulation, we assume the following values for this voltage

$V_{s}= \begin{cases}1.0 & 0 \leq t \leq T_{s} \\ 0.2 & T_{s} \leq t \leq T_{f} \\ 1.0 & T_{f \leq t \leq T}\end{cases}$ 
where $T_{s}$ is the time at which the voltage sag starts, while $T_{f}$ is the time at which the voltage sage restrain. $T$ is the time at which the measurements end up. Figure 11 shows such type of voltage signal.

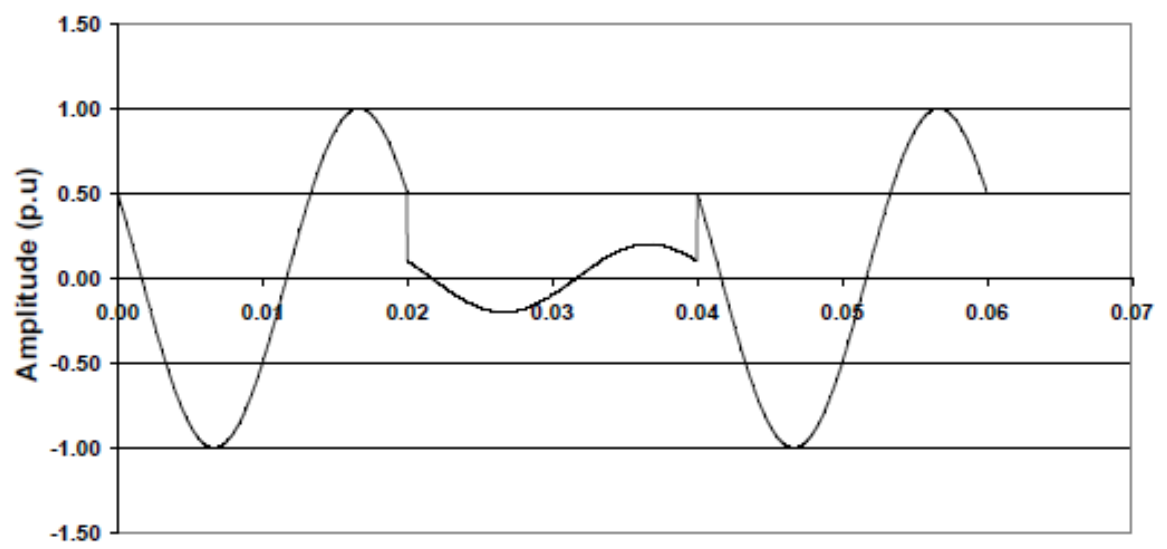

Time (s)

Figure 11. Voltage Sag

This signal is sampled at a sampling frequency of $40 \mathrm{kHz}$ with a one-cycle data window size for the three stages. The proposed technique, Kalman filter algorithm, is implemented to estimate the voltage magnitude as well as the phase angle.

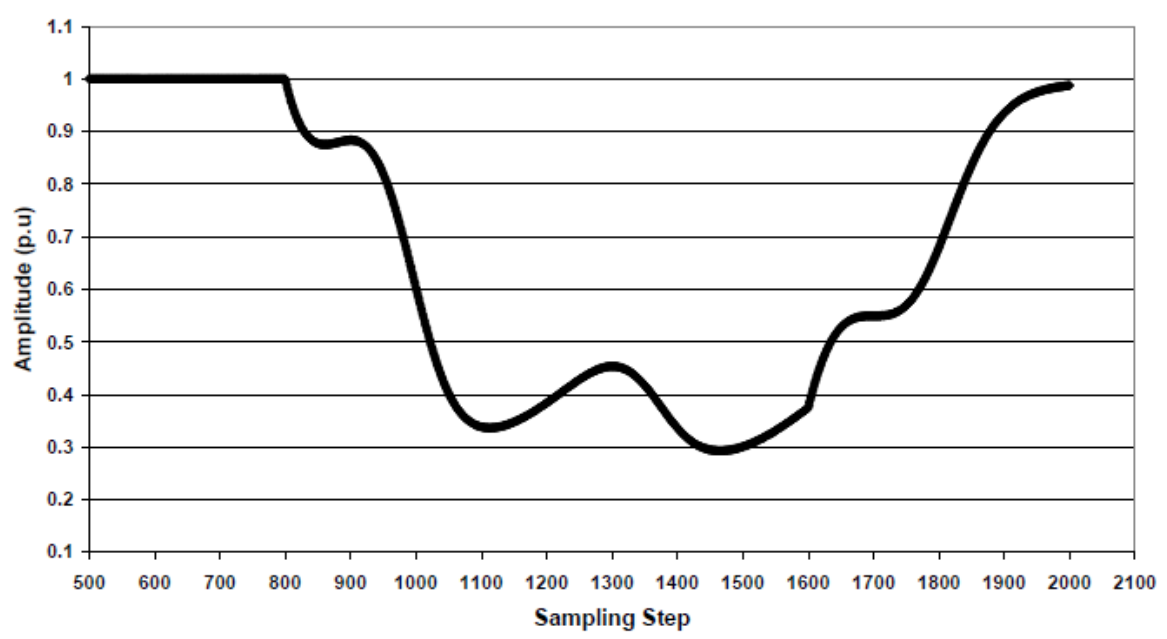

Figure 12. The estimated Voltage Amplitude (p.u) 
Figure 12 gives the estimated rms voltage during the three stages, while Figure 13 gives the estimated phase angle. Examining the two curves reveals the following remarks;

- The proposed algorithm estimates the voltage amplitude accurately in the first cycle and with a fair accuracy during the voltage sages and after the voltage sages

- One cycle data wind size during voltage sages is not enough to reach the actual voltage sages at least we need more than four cycles. This will be discussed in the next test

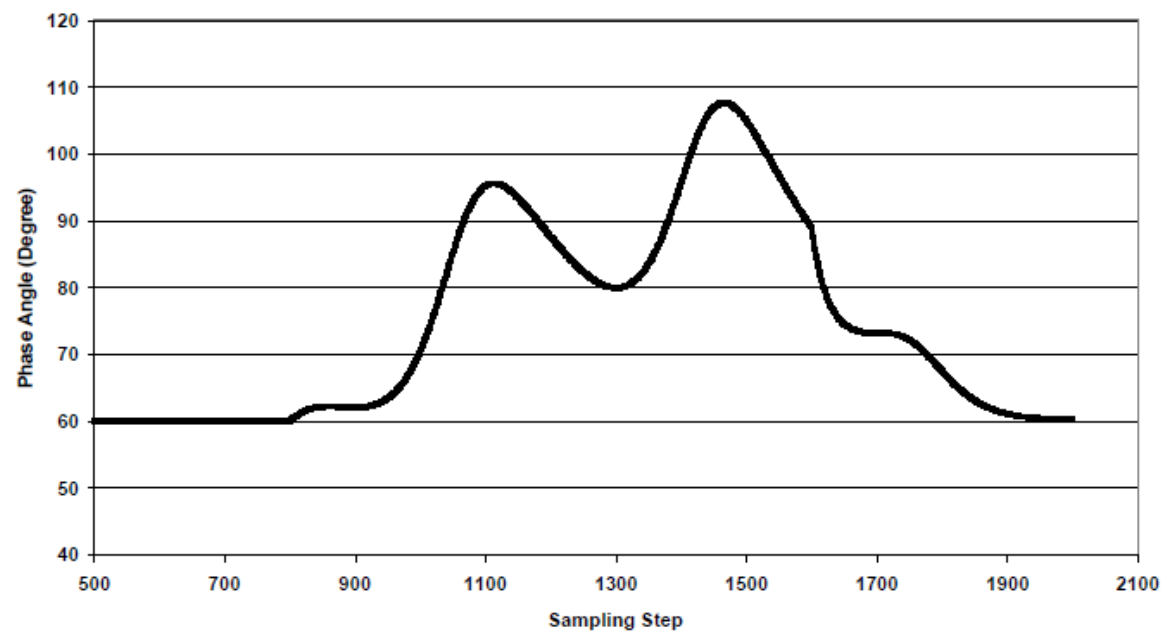

Figure 13. The estimated phase angle

- There is a phase jump during the voltage sags period, and the proposed algorithm estimates the phase angle accurately during pre voltage sages and after voltage sages.

Another test is conducted in the same voltage signal, where we increase the voltage sages' period to three cycles. Figure 14 gives such waveform

The estimated voltage amplitude and the phase angle are given in Figure 15, 16. Examining these two curves reveals that:

- Kalman filter is succeeded in identifying the period, where the voltage sags are occurred, as well as the amplitude of the voltage sags with an acceptable accuracy

- Increasing the number of cycles for the voltage sags makes the filter able to identify pre, during and after sags amplitudes with acceptable accuracy.

- It has been shown, as a common practice, that there is phase jump always occurs during the period of voltage sags

Another test is performed in this study, where we assume that the voltage signal contaminates with third and fifth harmonics with magnitude 0.25 and 0.125 respectively. Figure 17 gives the 


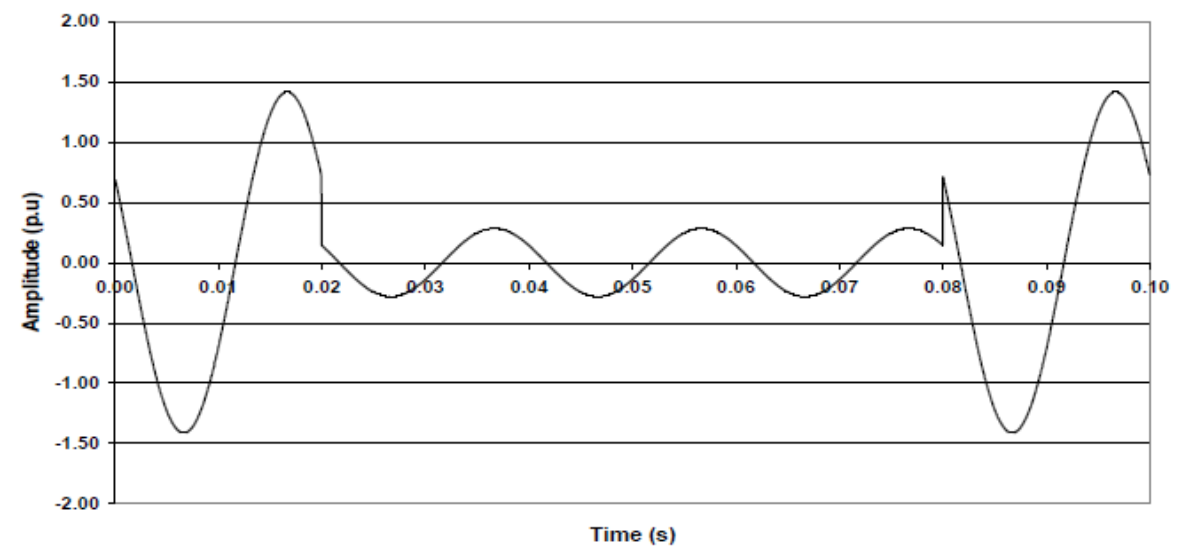

Figure 14. A Voltage sag

waveform of this voltage signal, while Figures 18, 19 give the estimated rms value of the fundamental component and its phase angle.

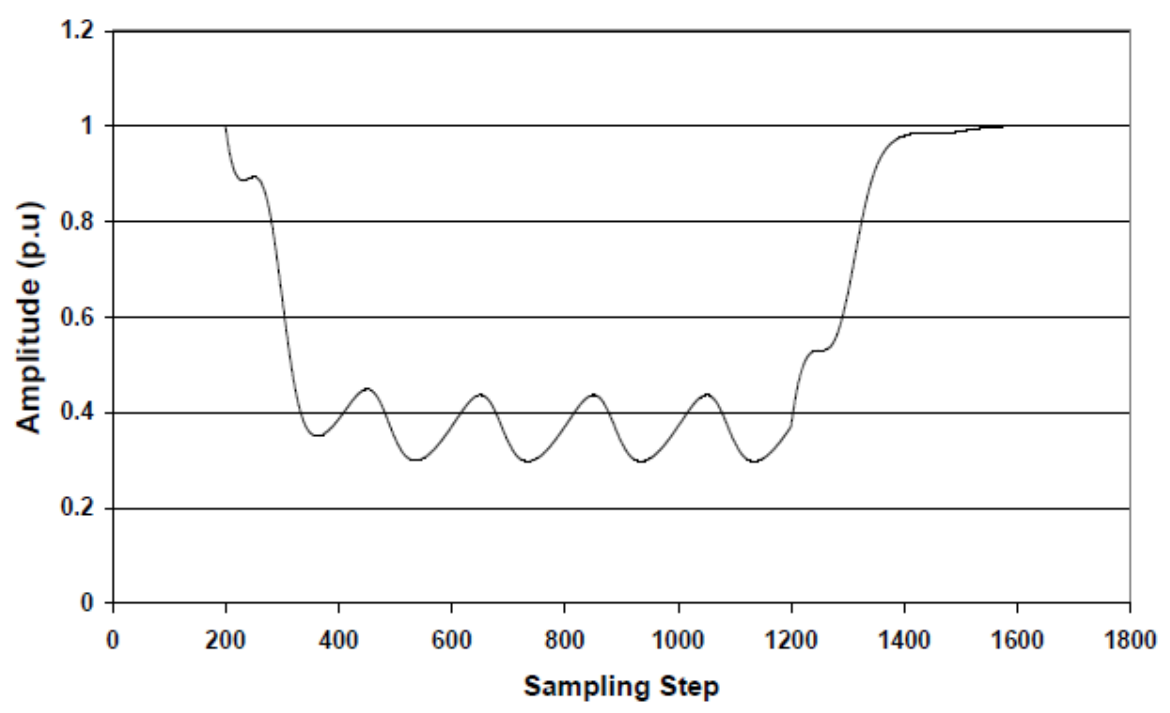

Figure 15. The estimated voltage amplitude 


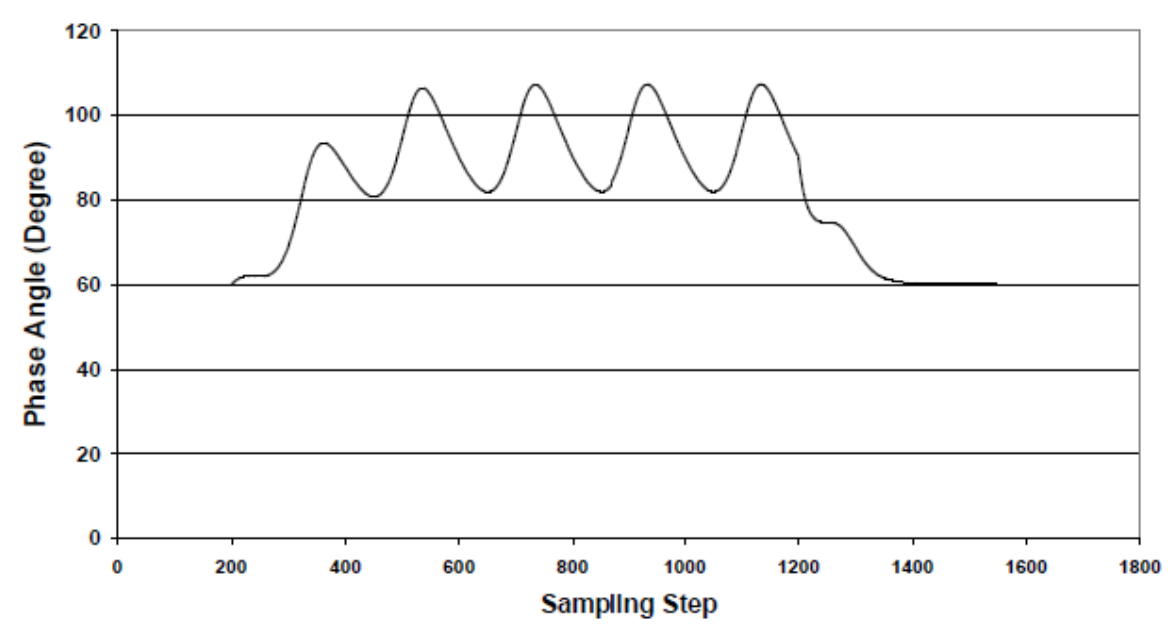

Figure 16. The Estimated Phase Angle

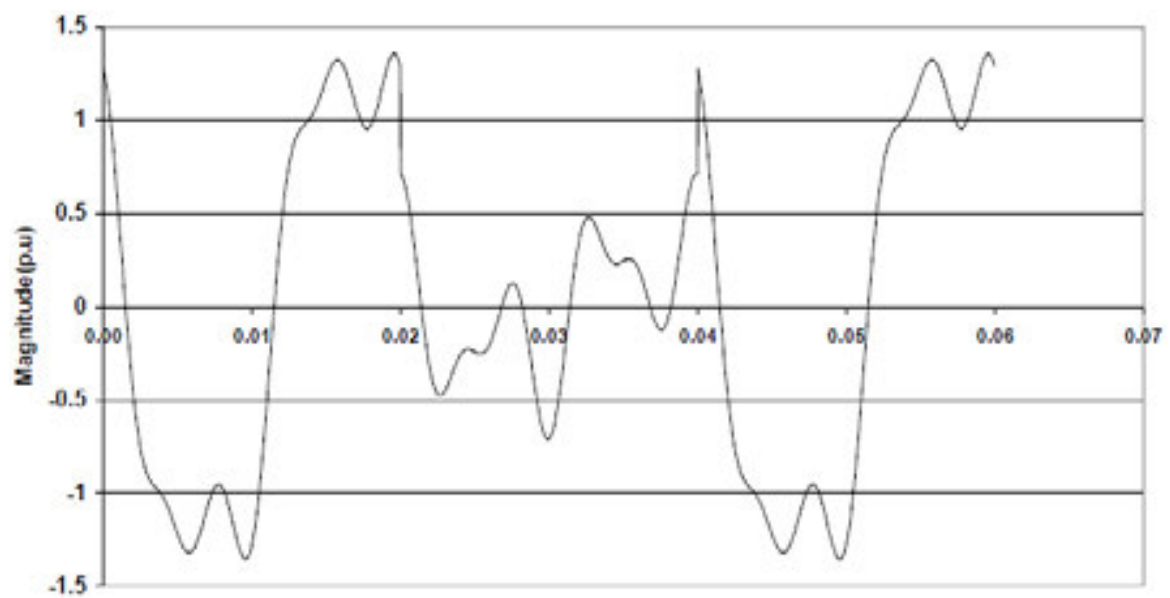

Sampling Step (k)

Figure 17. A Non-sinusoidal voltage experienced voltage sag 


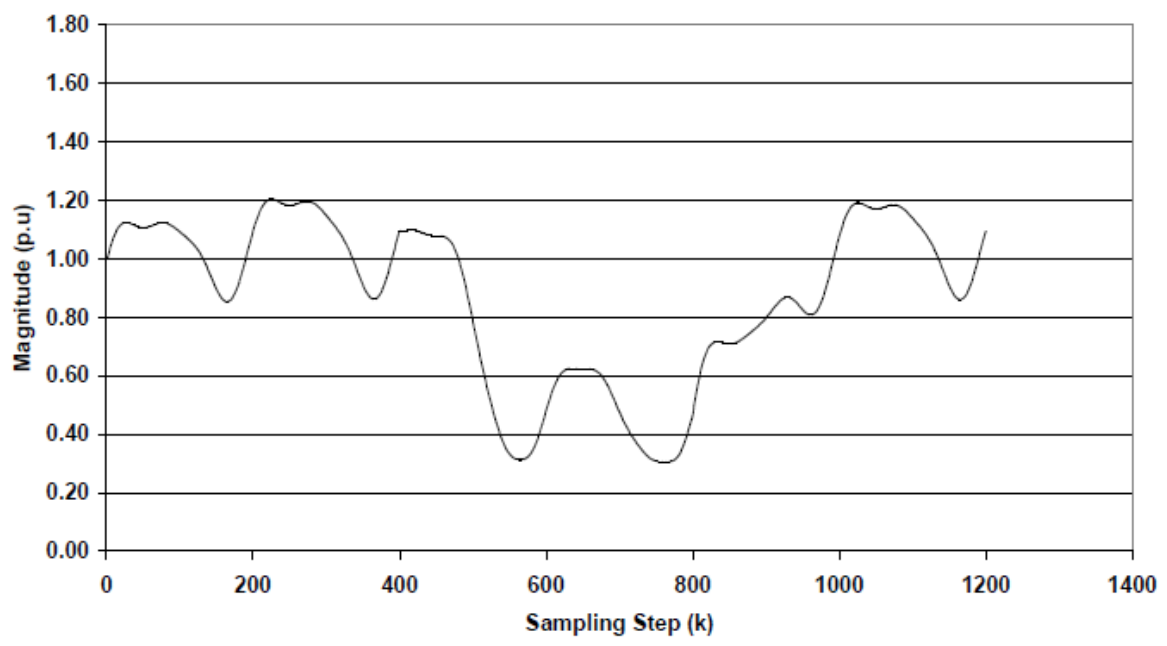

Figure 18. Estimated Fundamental Component

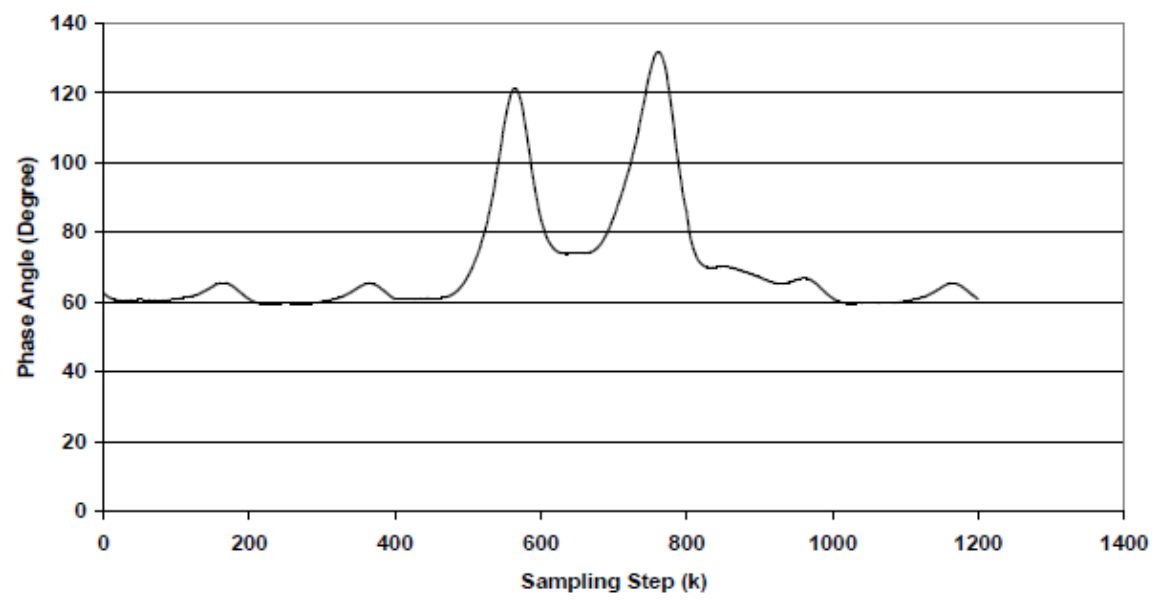

Figure 19. Estimated Fundamental Phase Angle

Examining these curves reveals that:

- Although the voltage signal is contaminated with harmonics, the proposed algorithm is succeeded to estimate the amplitude of the fundamental components in pre, during and after voltage sags with fair accuracy.

- The proposed technique estimates exactly the period of the voltage sags, 
- The phase angle jump during the voltage sags, in this case, is greater than that of nonharmonics contamination.

\subsection{Conclusions}

The main contributions of this section are:

- Kalman filtering algorithm is applied to estimate the harmonic components in a voltage signal of a power system. A full spectrum for identification of the fundamental component is not necessarily. Since the proposed algorithm estimates this component with a good accuracy.

- The proposed algorithm is succeeded in estimating the magnitude of the voltage signal that experienced voltage sag. Good estimates are produced for the voltage signal before, during and after the voltage sags.

- An accurate estimate for the phase angle and the phase angle jump is produced using the proposed algorithm.

- The proposed algorithm produces good estimate for the period of the voltage sags. The starting and ending time of the voltage sags is shown in the Figures within the text.

- The proposed Kalman filtering technique does not affect by the number of samples for harmonics measurements. A small data window size can provide all the information for harmonics components. Furthermore, the sampling frequency has no effect on the estimated components. Providing that the sampling theorem be satisfied.

\section{Parks' transformation}

Park's transformation is a well known transformation used in the analysis of electric machines, where the three rotating phases abc are transferred to three equivalent stationary dq0 phases (d-q reference frame). In this section this transformation is implemented to recognizing and classifying the power quality events, either for three-phase or single phase circuits. The proposed algorithm transferred the utility signal to a complex phasor. The magnitude of this phasor depends on the magnitude of the utility signal either a three-phase or a single phase signal. This technique produces the complex phasor loci that depend on the power quality event; voltage sags, voltage flickers, voltage swell and harmonics. The time of starting the disturbance is chosen randomly and the length of disturbance is arbitrary. Implementation of this technique is succeeded in recognizing and classifying the power quality events. Simulated results are presented, for three-phase and single phase events. wi

\subsection{Identification processes}

In the following steps we assume that $\mathrm{m}$ samples of the three phase currents or voltage are available at a pre-selected sampling frequency that satisfying the sampling theorem. 
The forward transformation matrix

$$
P=\sqrt{\frac{2}{3}}\left[\begin{array}{ccc}
\sin \omega t & \sin (\omega t+120) & \sin (\omega t+240) \\
\cos \omega t & \cos (\omega t+120) & \cos (\omega t+240) \\
\frac{1}{\sqrt{2}} & \frac{1}{\sqrt{2}} & \frac{1}{\sqrt{2}}
\end{array}\right]
$$

The matrix given in equation (17) can be computed off line if the frequency of the voltage and/ or current signals as well as the sampling frequency and the number of samples are known in advance. If the matrix given in equation (17) is multiplied digitally by the samples of the threephase voltage that are sampled at the same sampling frequency of matrix (17), a new set of three -phase samples are obtained, we call this set a $d q o$ set (reference frame). If we use only the samples for the two perpendicular phases $\mathrm{d}$ and $\mathrm{q}$, then the resulting phasor at a sample $k$ is given by;

$$
V(k)=V_{d}(k)+j V_{q}(k)
$$

The magnitude of this phasor is given by

$$
V(k)=\sqrt{V_{d}^{2}(k)+V_{q}^{2}(k)}
$$

While the phase angle is given by

$$
\phi(k)=\tan ^{-1} \frac{V_{q}(k)}{V_{d}(k)}=\omega k \Delta T+\delta(k)
$$

In this study we may assume, during the power quality events, that the frequency of the voltage signal is constant and equals to the nominal frequency of the system, 50/60 Hz. However if this frequency is not constant, it can be calculated from equation (20) using the least error squares estimation algorithm. Equation (19) is a good indicator to the status of the system signal. Where $V(k)$ is plotted with against the time, and every event has a form that different from other events. If the relation between $V_{d}(k)$ and $V_{q}(k)$ a loci is produced that describes the event. In the next section a computer simulation is carried out for different types of power quality events. 


\subsection{Computer simulation}

In this study we assume that the voltage signal frequency is constant at $50 \mathrm{~Hz}$ during the events, and a sampling frequency of $10000 \mathrm{~Hz}$ is used $((\Delta T=0.1 \mathrm{~ms}), 200$ samples per cycle and 500 samples are used. Before the event 200 samples, 1cycles, are used as pre-estimated period and 100 samples, 0.5 cycles as the event period, and finally 200 samples, 1 cycles, are used as after event period. Different types of power quality events are simulated, these including voltage flickers, voltage sags, voltage swell, momentary interruption and finally voltage harmonics.

\subsubsection{Voltage sags}

In the first part of this study we assume that one of the phases has only voltage sag, where the voltage is dropped from 1 p.u to 0.25 p.u, single- phase sag. While in the second study, the three phases have the same amount of sags, where the voltage in the three phases is dropped from 1.0 p.u to 0.25 p.u. Figure 20 to 22 gives the results obtained for the single phase sag.

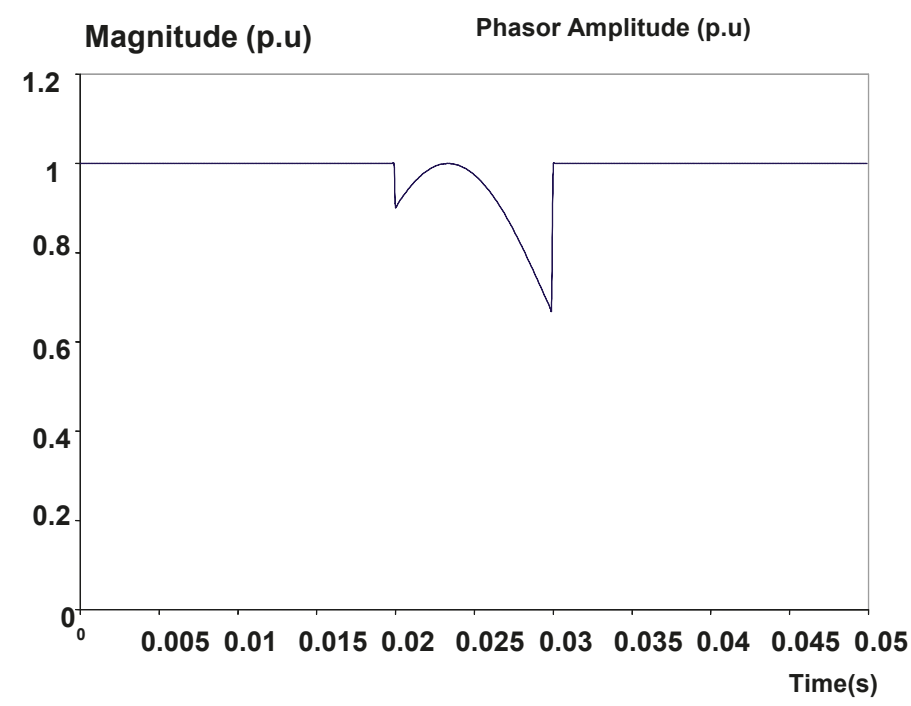

Figure 20. Variation of the phasor magnitude Single Phase voltage sags, 


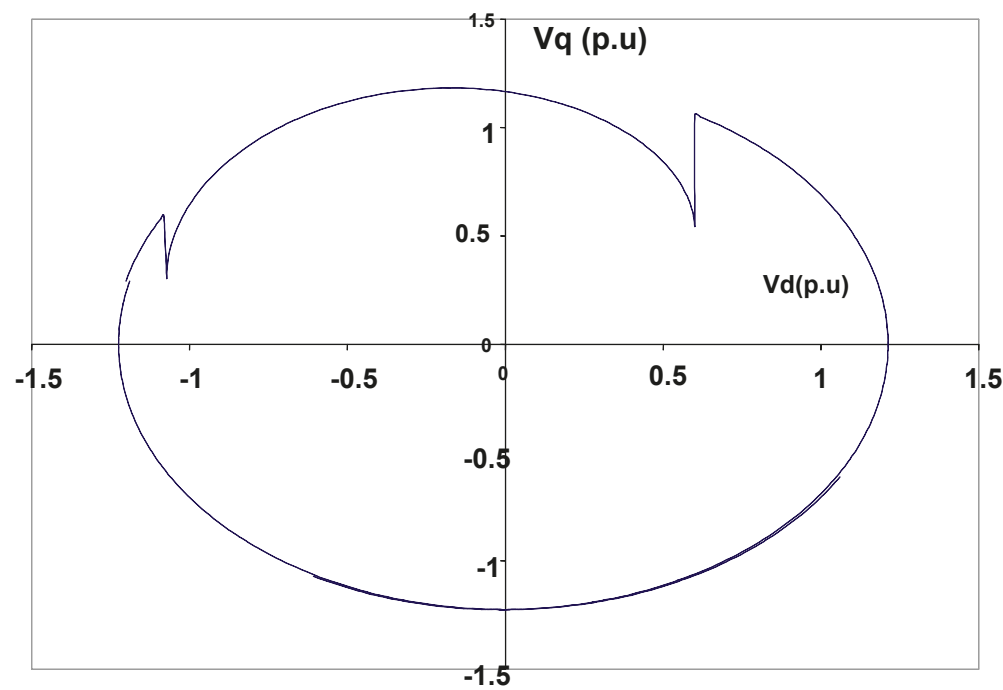

Figure 21. Locus of $V_{d}$ and $V_{q}$ for a single phase sag

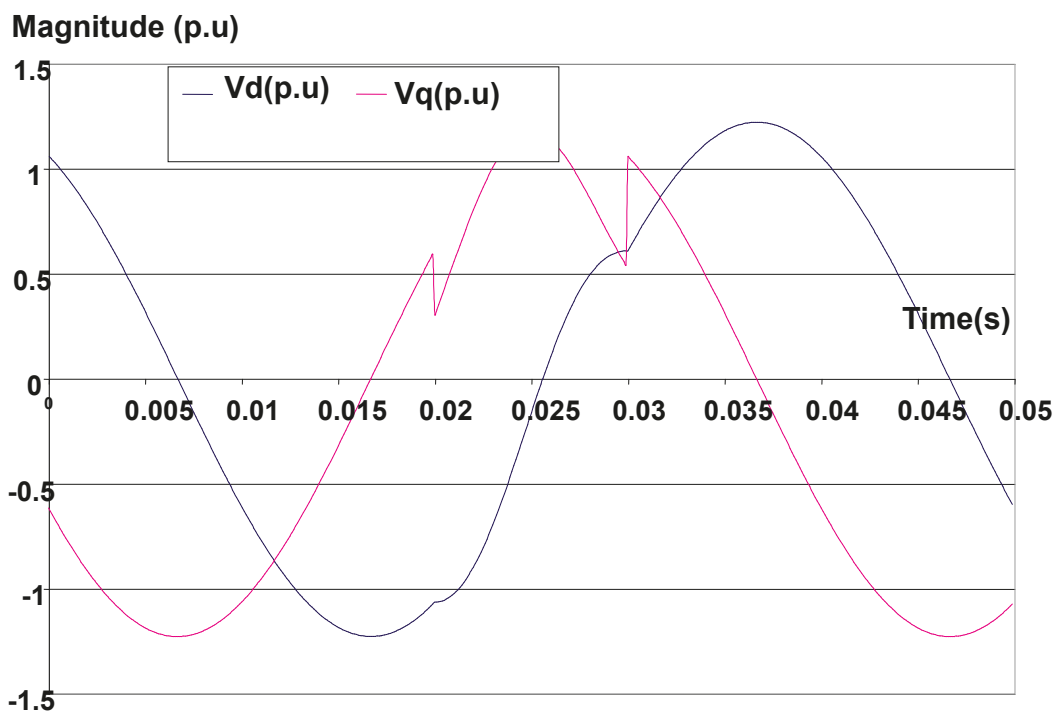

Figure 22. Waveforms for $V_{d}$ and $V_{q}$ for single phase sag. 


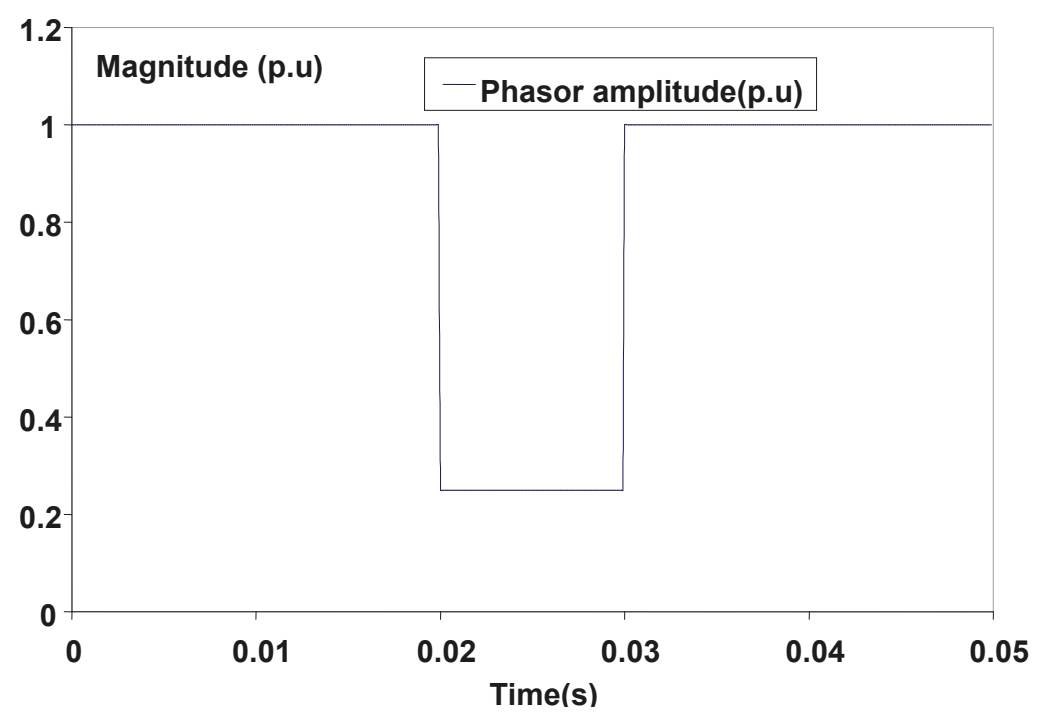

Figure 23. Variation of the phasor magnitude three- Phase voltage sags,

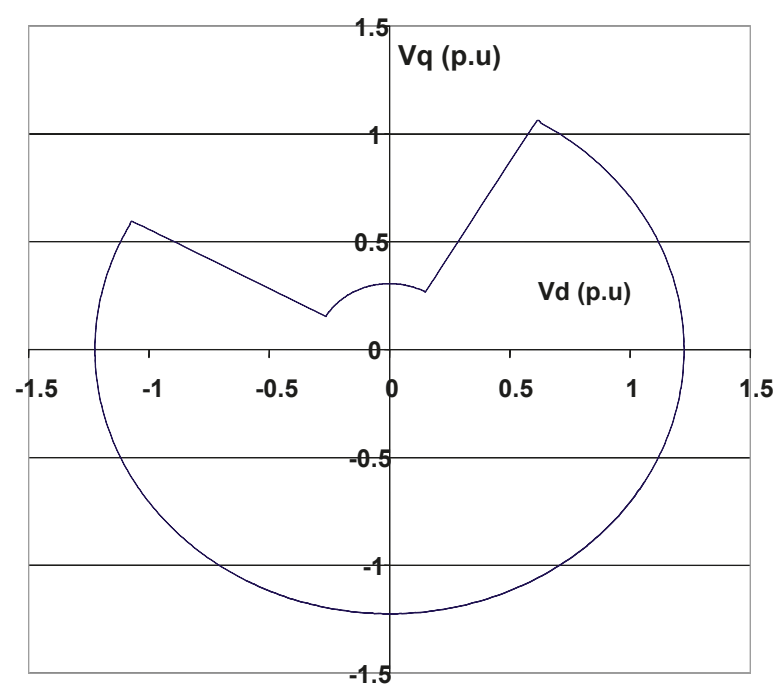

Figure 24. Locus of $V_{d}$ and $V_{q}$ for three- phase sag 


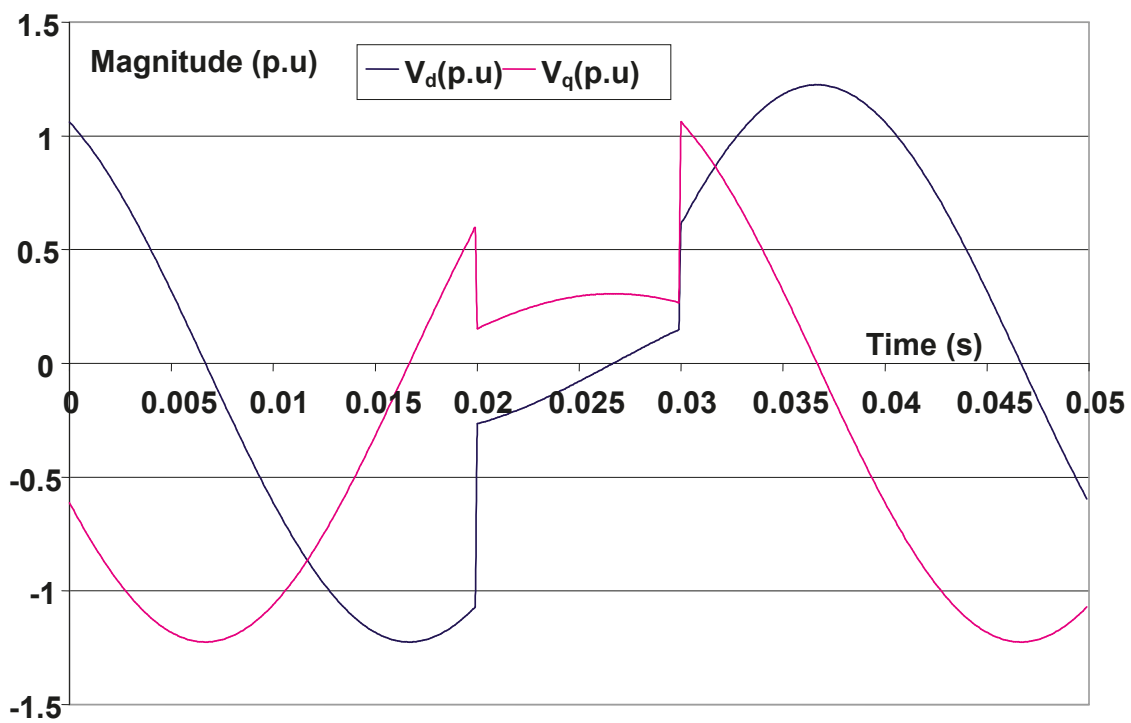

Figure 25. Waveforms for $V_{d}$ and $V_{q}$ for three-phase sag.

Examining these figures reveals:

- For the single phase sage, during the sage period the rms value of the voltage is dropped to a value that does not equal to the voltage magnitude at that period, but it does for three -phase voltage sag. Figures 20 and 26. experienced a voltage sage, while in the This is due to the nature of the transformation, where in the single pahse sags one of the phases is only three phase, the three phases are experienced the voltage sags. It does mean that during the sage period, for single phase, two phases are balanced while the third one is distorted, while in the three- phase the three phases are distorted. This is clearly indicated in Figures 21 and 23

- Figures 22 and 25 indicate that the variation of the two voltages $V_{d}$ and $V_{q}$ in the time domain is not the same for single phase sags, but they are the same for the three phases. In the single phase $V_{q}$ is a little bit distorted during the sag period while for three -phase sag $V_{d}$ and $V_{q}$ are large distorted during the sag period.

\subsubsection{Voltage flicker}

In the first part of this study we assume that one of the phases has only voltage flicker, single phase flicker. While in the second study, the three phases are experienced the same voltage flicker, Figure 26 to 9 gives the results obtained for the single phase flicker while Figures from 19 to 21 give the results obtained for the three phase voltage flicker, where the three phases are assumed to experience the same voltage flicker. Examining these curves reveals the following remarks: 
- The phasor amplitude in figure 26 has an amplitude modulation during the data window size

- The locus of $\mathrm{V}_{\mathrm{d}}$ and $\mathrm{V}_{\mathrm{q}}$ in Figure 27 is not a pure circle as it should be, but it is an ellipse with distorted amplitude.

- The signals waveform for the two voltages $\mathrm{V}_{\mathrm{d}}$ and $\mathrm{V}_{\mathrm{q}}$ are distorted signals.

Indeed looking to Figure 26, one can notice that the system experiences a voltage flicker

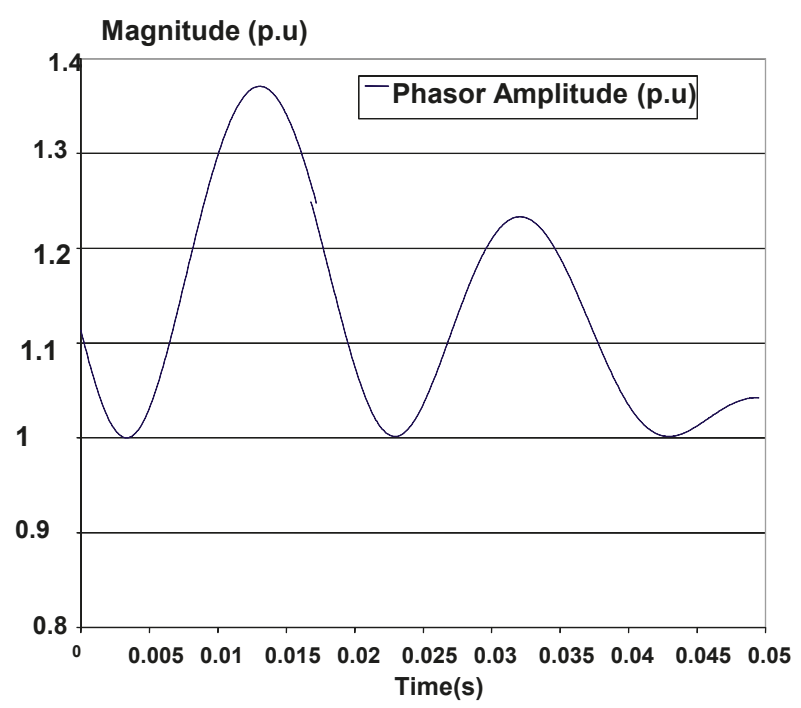

Figure 26. The phasor voltage in the Time domain for a single phase flicker

Examining figures 29 to 31, one can conclude the following remarks:

- The phasor amplitude decreases down during the data window size, and looking to this figure one can notice that the system experiences voltage flicker.

- The locus of $\mathrm{V}_{\mathrm{d}}$ and $\mathrm{V}_{\mathrm{q}}$ is an open ellipse despite of the single phase voltage flicker where this locus is a distorted closed ellipse

- The wave form of the voltage $\mathrm{V}_{\mathrm{d}}$ and $\mathrm{V}_{\mathrm{q}}$ is an amplitude modulated wave.

General speaking, for voltage flicker, the amplitude of the transformed signals is demodulated amplitude with amplitude greater than one per unit. 


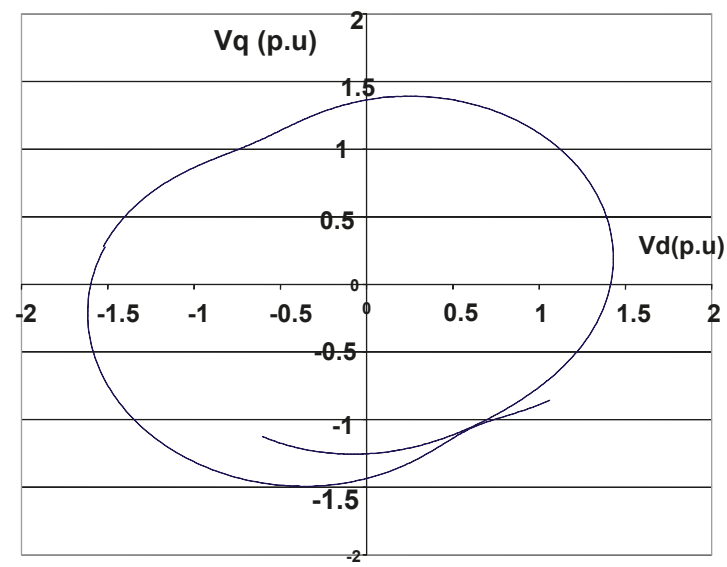

Figure 27. Locus of $V_{d}$ and $V_{q}$ for a single phase flicker

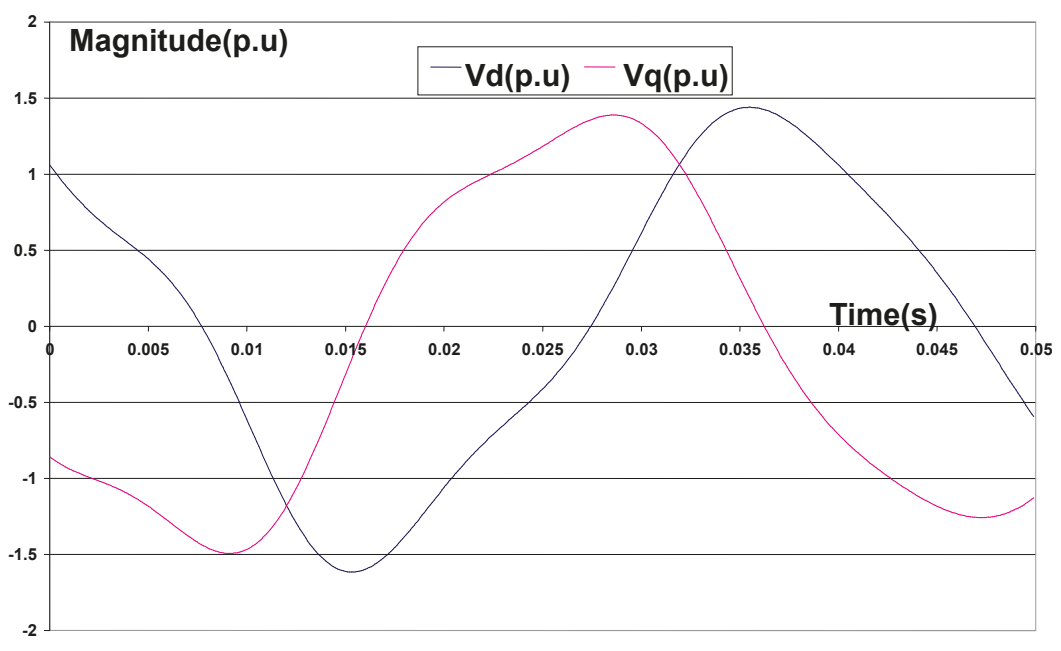

Figure 28. $V_{d}$ and $V_{q}$ in the time domain for a single phase flicker 


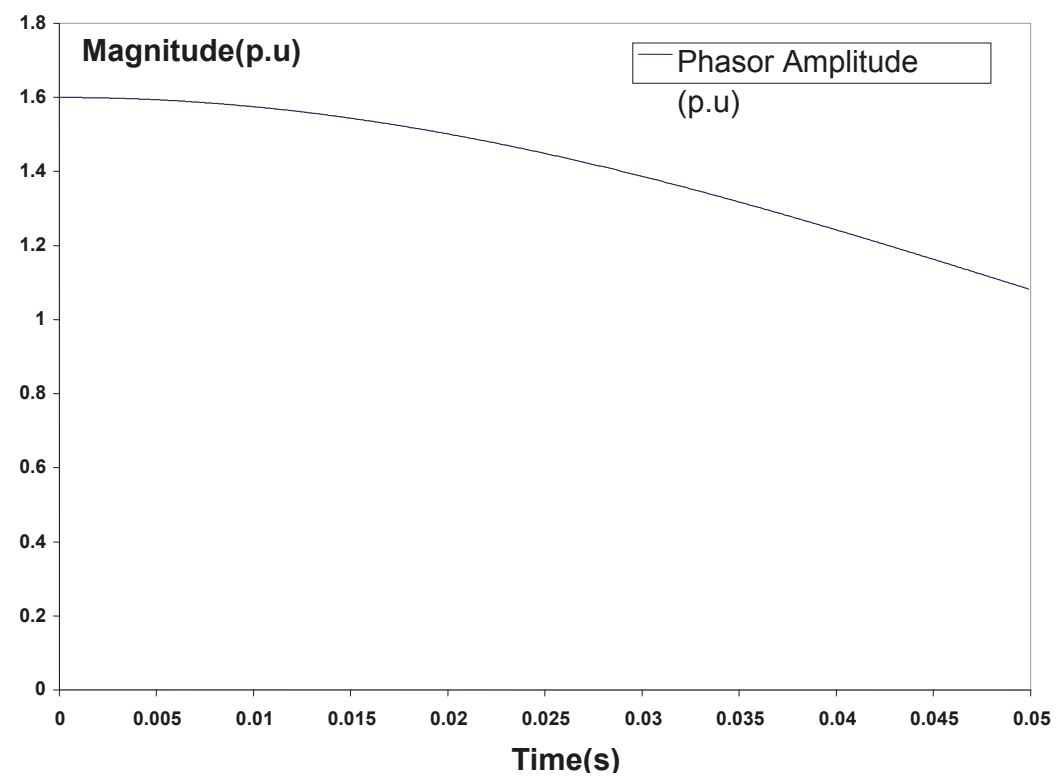

Figure 29. Phasor magnitudes in the time domain for a three phase flicker

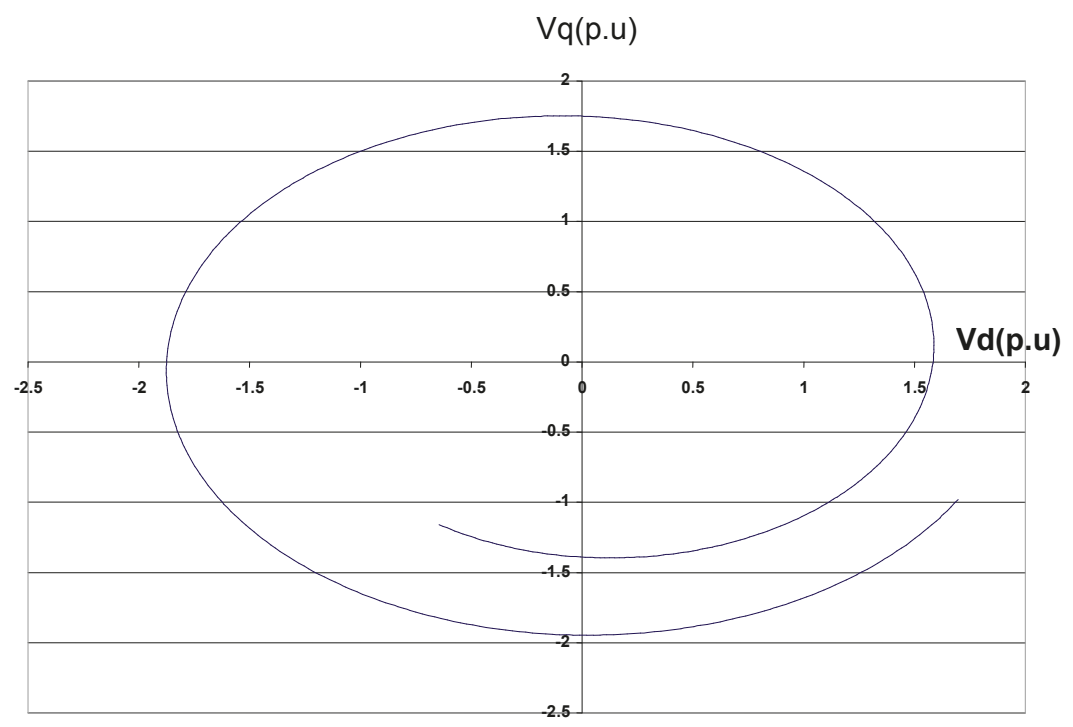

Figure 30. Locus $V_{d}$ and $V_{q}$ for a three phase flicker 


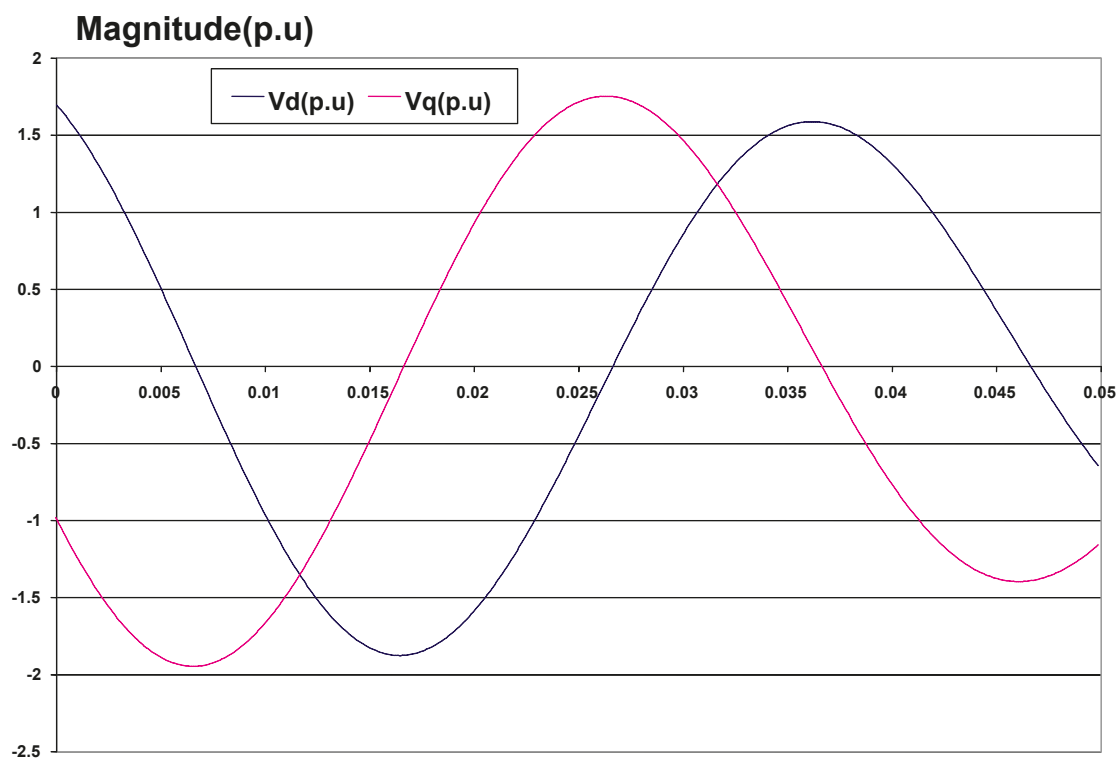

Time(s)

Figure 31. $V_{d}$ and $V_{q}$ in the time domain for a three-phase flicker

\subsubsection{Voltage swell}

In this test a single phase swell is implemented on the voltage signal, where the voltage is increased 50 percent more for a short period, about $10 \mathrm{~ms}$. Figures 32, 33 and 34 give the results obtained. Examining these figures one can reveal the following remarks:

- Examining Figure 32, the phasor amplitude equal to 1 per unit in the pre-swell period, then it increases during swell period and then comes back again to a value of one per unit.

- The locus of $\mathrm{V}_{\mathrm{d}}$ and $\mathrm{V}_{\mathrm{q}}$ is not a pure circle, but it is a distorted circle during the time of voltage swell.

- The signal for $\mathrm{V}_{\mathrm{d}}$ and $\mathrm{V}_{\mathrm{q}}$ is not pure sinusoids, especially during the time of swell.

In the second part of the test an equal swell is implemented for the three phase signals, and $\mathrm{V}_{\mathrm{d}}$ and $\mathrm{V}_{\mathrm{q}}$ are calculated. Figures 35, 36 and 37 give the results obtained. Examining these figures reveals the following

- Looking to Figure 35, immediately one can notice that the voltage has a swell with a magnitude of 1.5 p.u during the swell period

- The locus of $\mathrm{V}_{\mathrm{d}}$ and $\mathrm{V}_{\mathrm{q}}$ is not a pure circle, but it is a distorted circle during the time of voltage swell. 


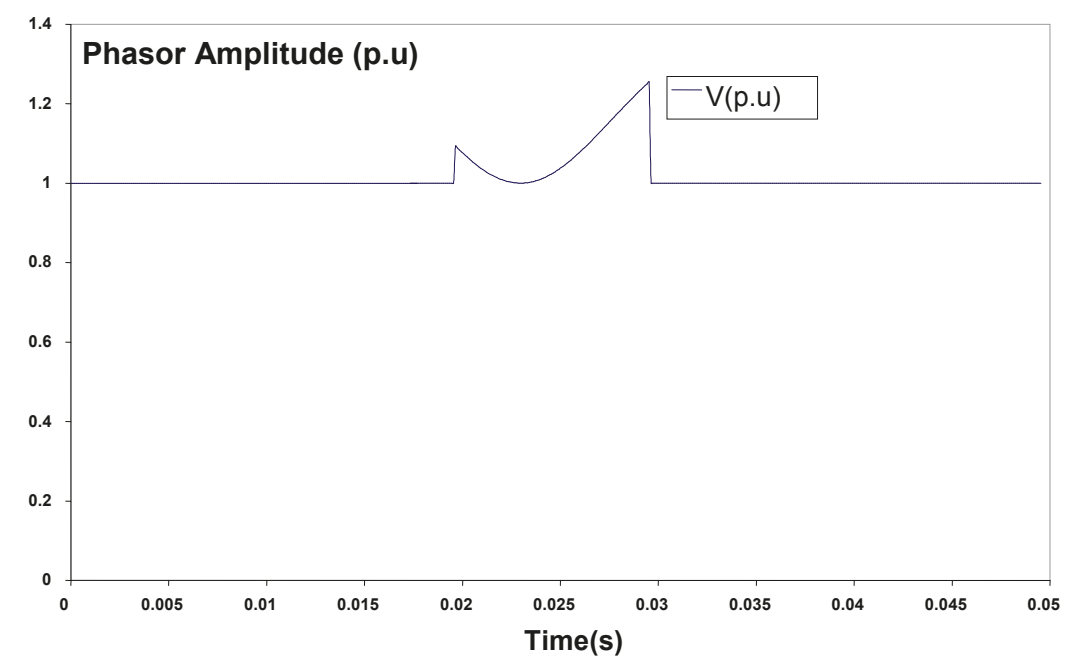

Figure 32. Phasor Magnitude for single phase swells

- The signal for $\mathrm{V}_{\mathrm{d}}$ and $\mathrm{V}_{\mathrm{q}}$ is not pure sinusoids, especially during the time of swell.

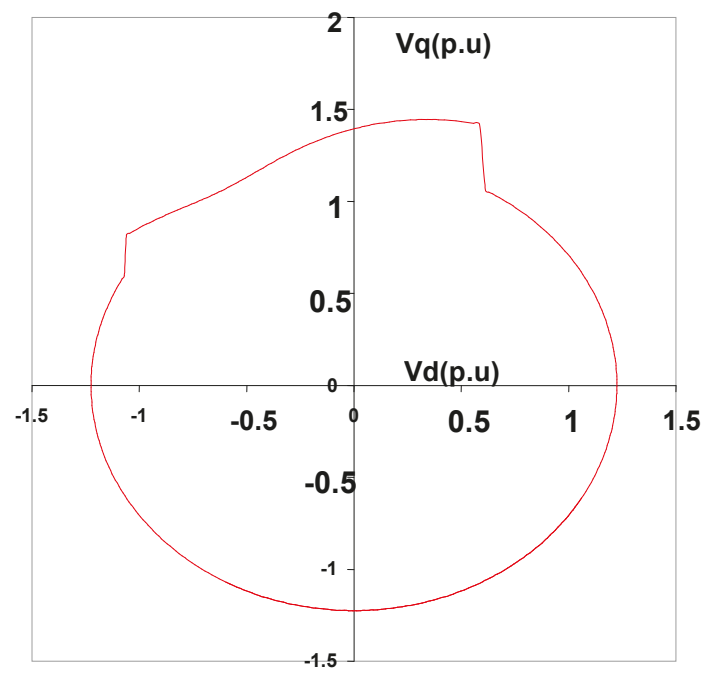

Figure 33. Locus diagram for a single phase swell 


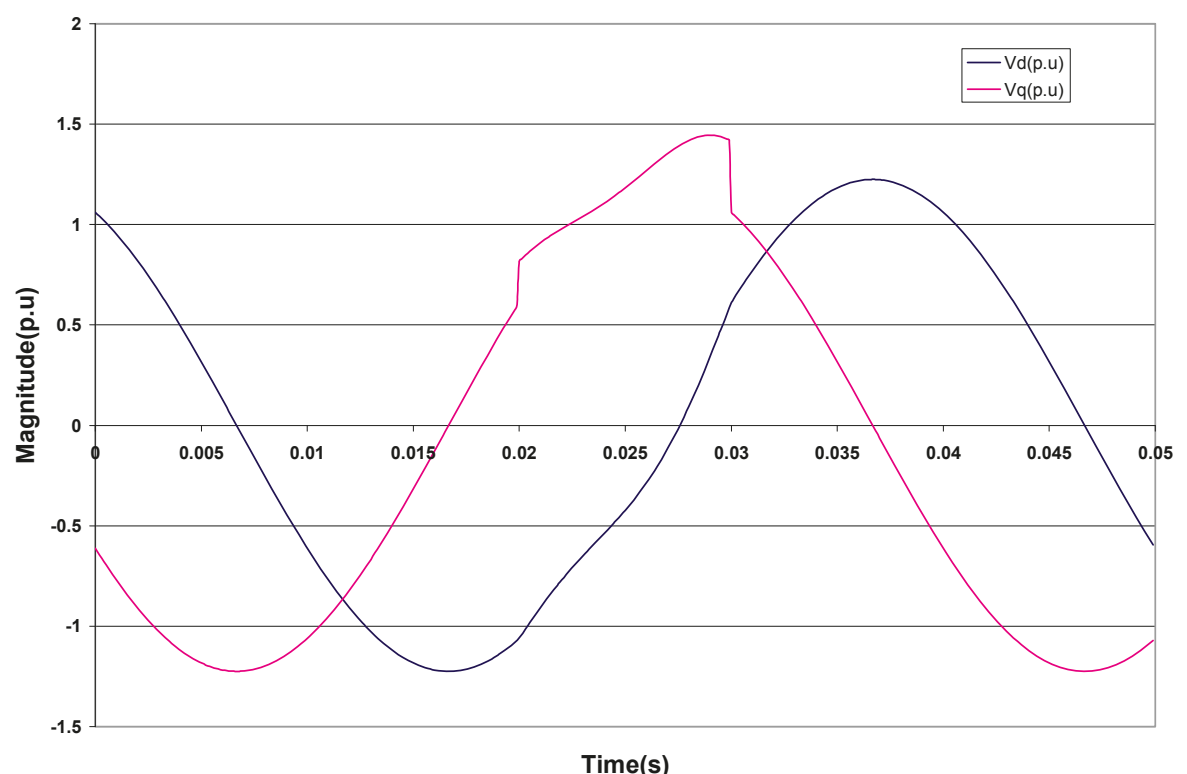

Figure 34. $V_{d}$ and $V_{q}$ in the time domain for a single phase swell

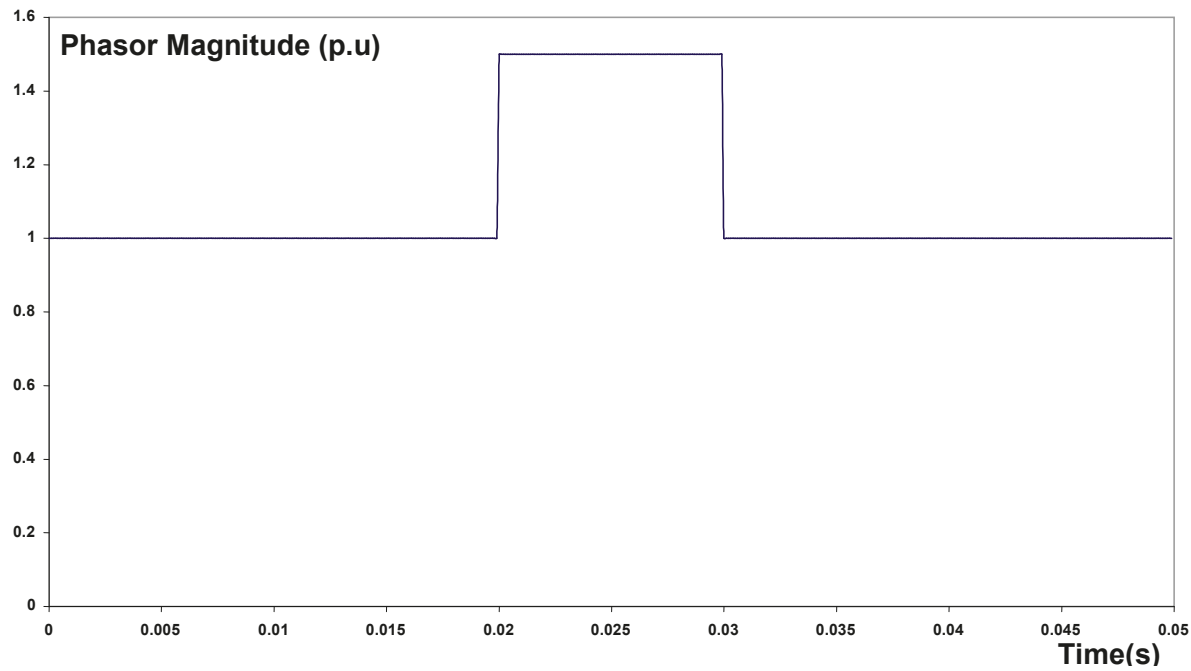

Figure 35. Phasor magnitudes in the time domain for a three phase swell 


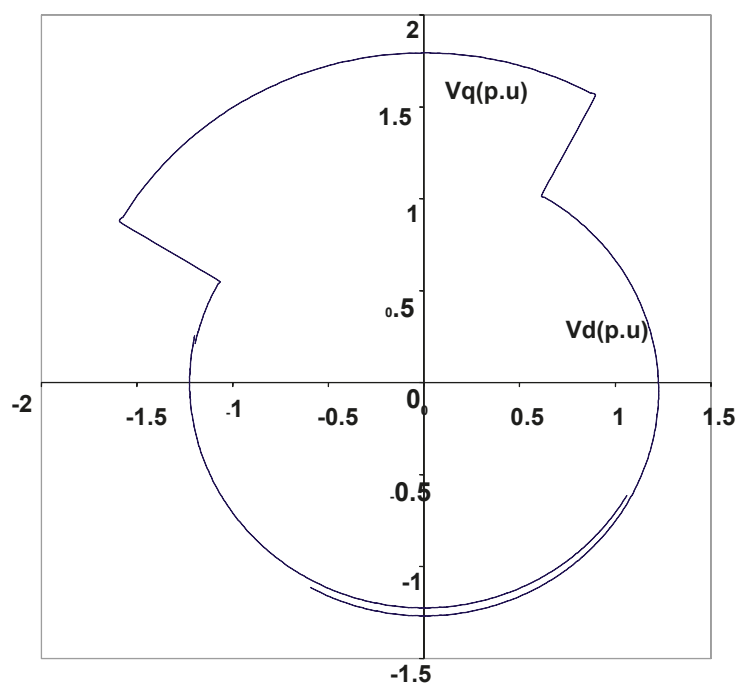

Figure 36. Locus diagram for a three phase swell

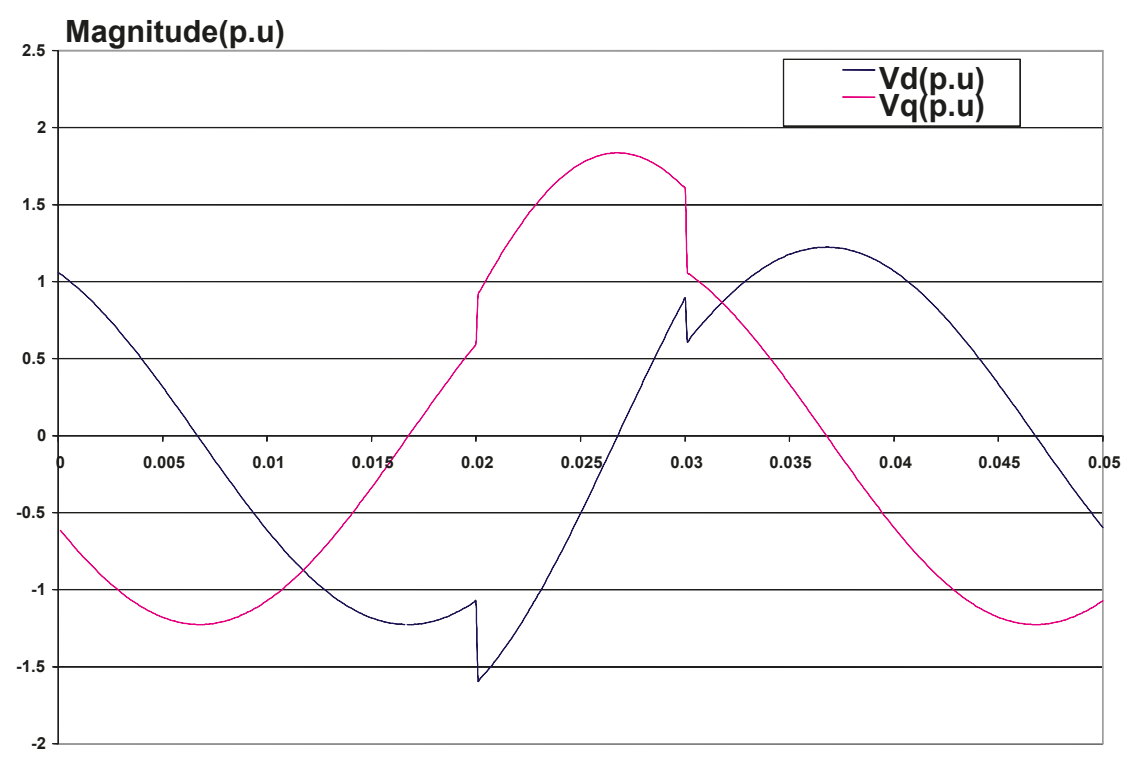

Time (s)

Figure 37. $V_{d}$ and $V_{q}$ in the time domain for a three phase swell 


\subsubsection{Voltage harmonics}

In this test the voltage signal is contaminated with third harmonics only. In the first part of the test we assume that one phase is only contaminated with harmonics, while the other two phases are not. Figures 38 to 40 gives the results obtained. Examining this curves one can notice the following:

- The phase magnitude in the time domain is a time varying magnitude and it is not a pure sinusoidal.

- The locus diagram is a distorted locus not a pure circle as it should be for uncontaminated phase.

- The voltages $\mathrm{V}_{\mathrm{d}}$ and $\mathrm{V}_{\mathrm{q}}$ in the time domain are not sinusoidal signals, but they are distorted.

In the second part of this test, we assume that the three phases are contaminated with the same order of harmonics, a balanced three phase harmonics contaminated system. Figures 41 to 43 give the results obtained. Examining these curves reveals the following remarks:

- Phasor magnitudes in the time domain for three- phase harmonics contamination is a pure sinusoidal with amplitude greater than one per unit, maximum value is 1.2447 per unit and the minimum value is 0.75 per unit and the average value between the two peaks is about one per unit.

- The locus diagram for three- phase harmonics contamination is a symmetrical shape about both axes

- $\mathrm{Vd}$ and $\mathrm{Vq}$ in the time domain for three- phase harmonics contamination are harmonics contaminated signals, distorted signals

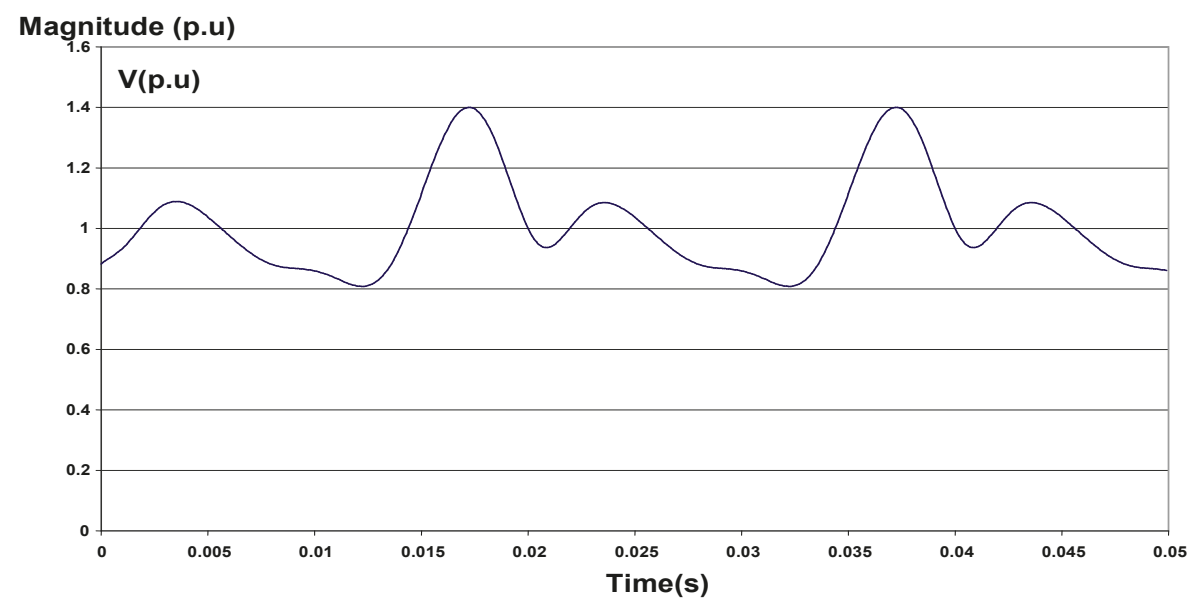

Figure 38. Phasor magnitudes in the time domain for a single phase harmonics contamination 


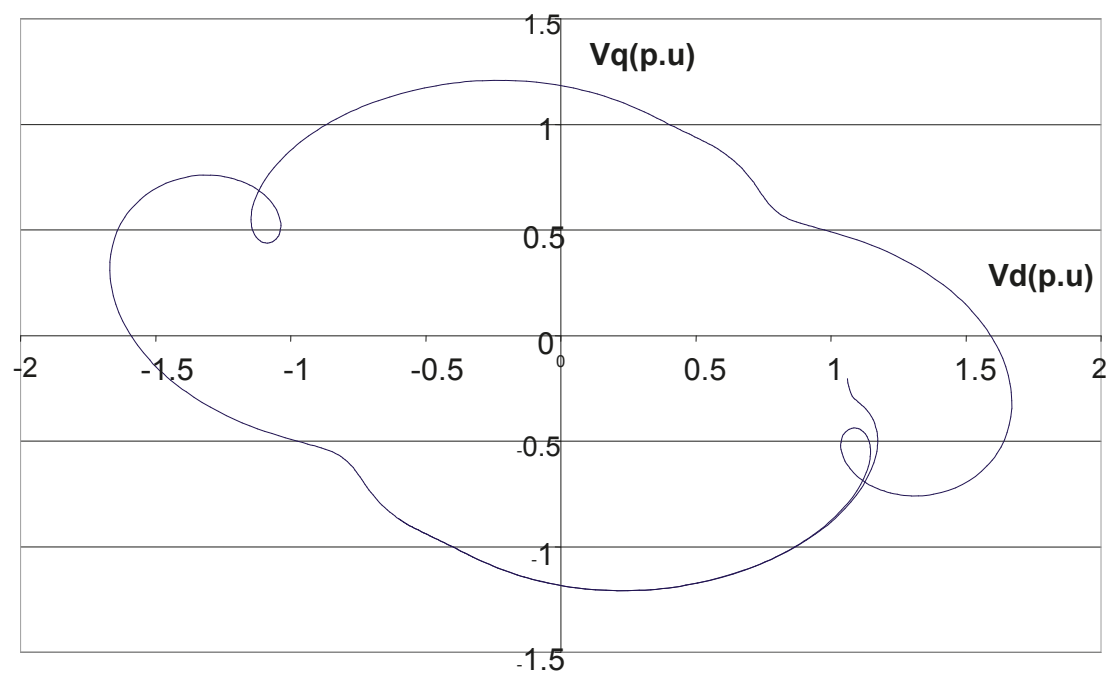

Figure 39. Locus diagram for a single phase harmonics contamination

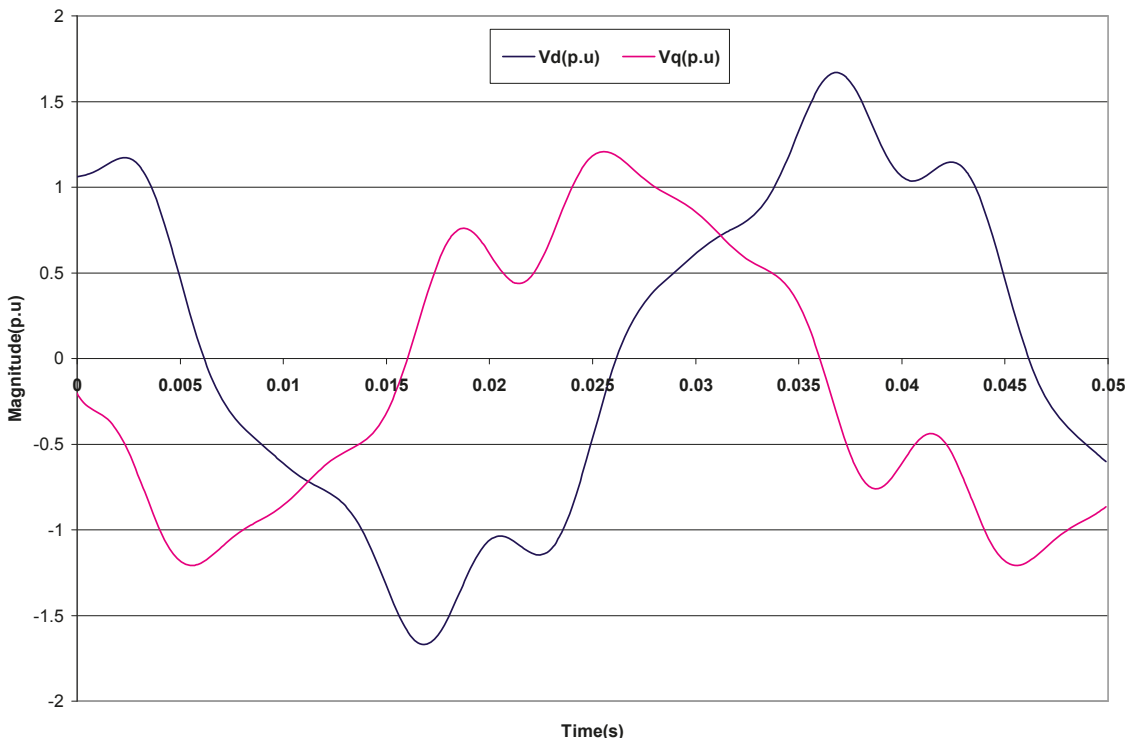

Figure 40. $V_{d}$ and $V_{q}$ in the time domain for a single phase harmonics contamination 


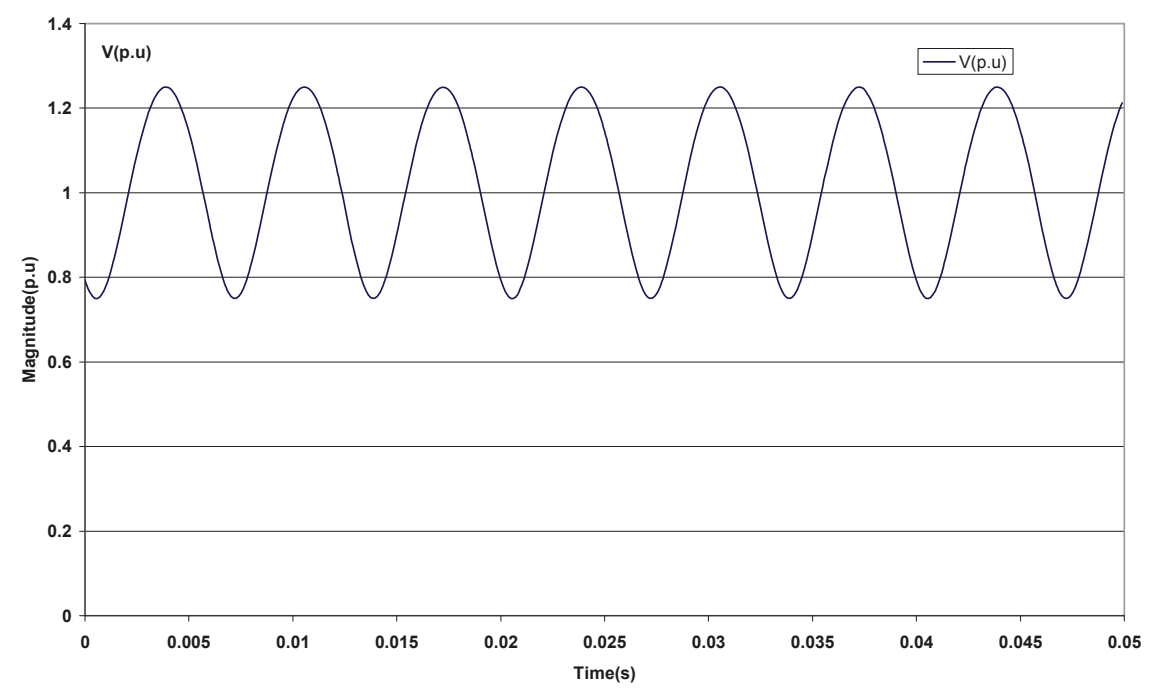

Figure 41. Phasor magnitudes in the time domain for three- phase harmonics contamination

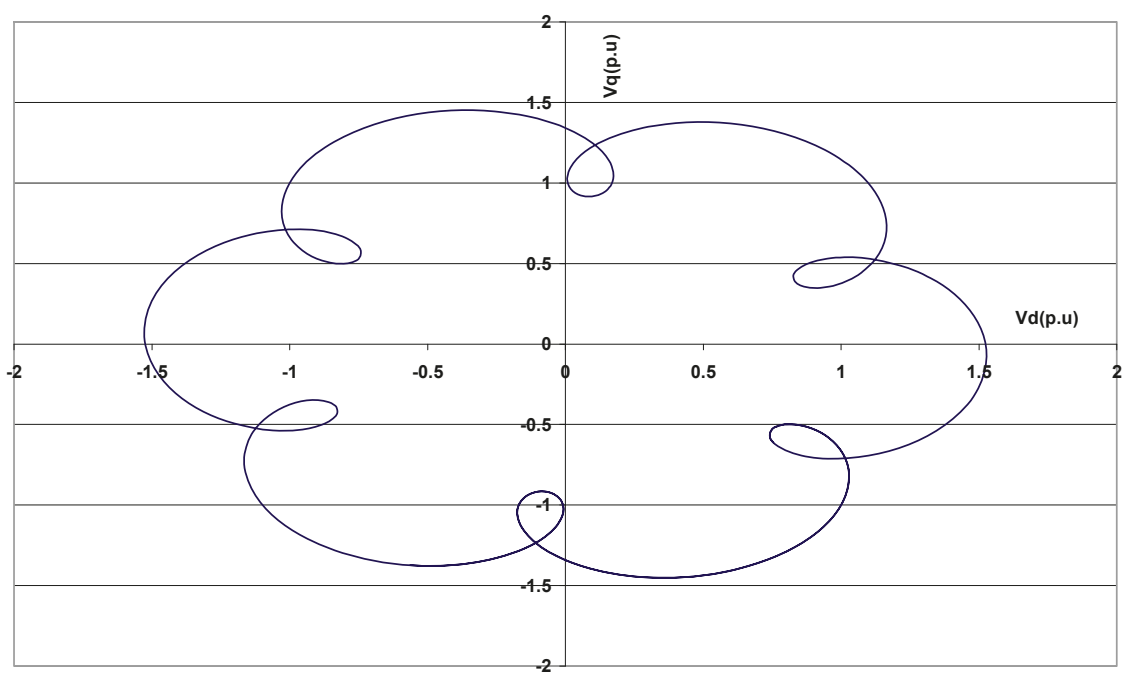

Figure 42. Locus diagram for three- phase harmonics contamination 


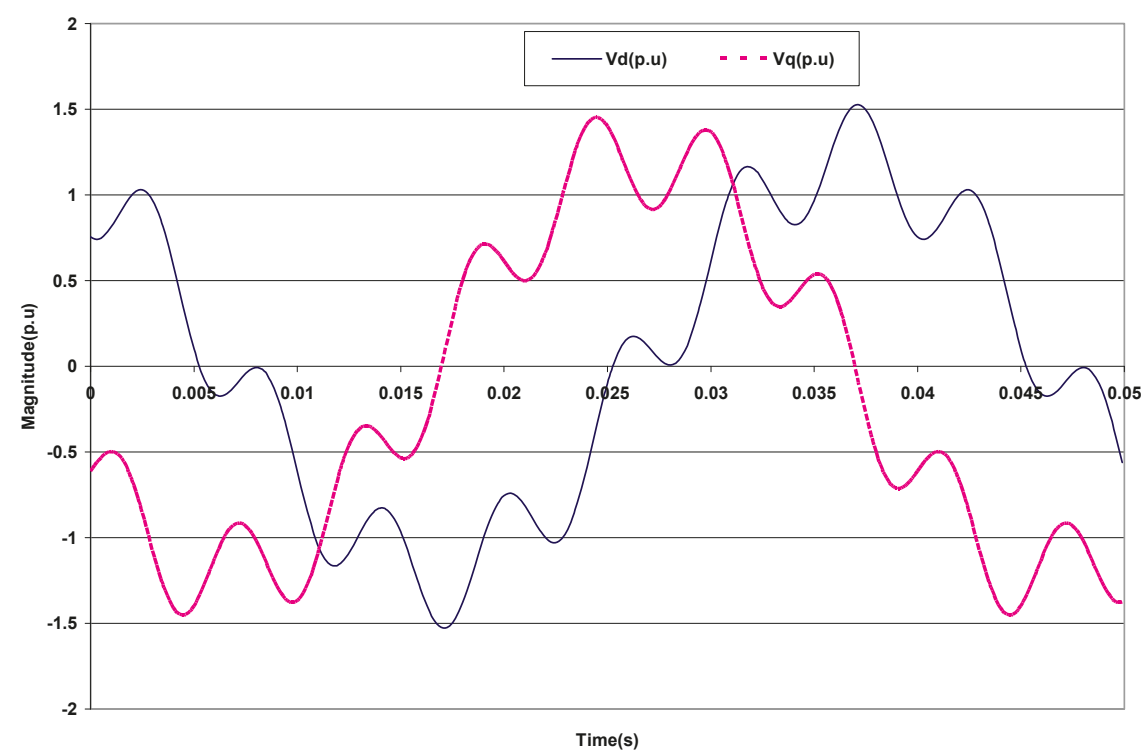

Figure 43. $V_{d}$ and $V_{q}$ in the time domain for three- phase harmonics contamination

\subsection{Conclusions}

The main contributions of this section are:

- Park's transformation is implemented to recognizing and classifying the power quality events; voltage sags, voltage flickers, voltage swell and harmonics, either for three-phase or single phase circuits.

- The proposed algorithm is succeeded in identification and classification of power quality events.

- The utility signal is transformed to a complex phasor. The magnitude of this phasor depends on the magnitude of the utility signal. The proposed technique produces the complex phasor loci that depend on the power quality event. Computer simulation results are presented within the text, these results indicate that the proposed technique is efficient, and one can recognize the type of event just by looking to the locus diagram of the two voltages $\mathrm{V}_{\mathrm{d}}$ and $\mathrm{V}_{\mathrm{q}}$.

- No metering instruments are needed to measure these events, since it is hard to find an instrument that can be used to measure such events.

- Classifying these events can help in making a decision to install the suitable power conditioning devices to suppress these events or part of them.

- It has been shown that the starting time of disturbance as well as the length of the disturbance has no effect on the Park's phasor. 


\section{Author details}

Soliman Abdel-Hady Soliman ${ }^{1}$ and Rashid Abdel-Kader Alammari²

1 Misr University of Science and Technology, Electrical Power and Machines Department, Giza, Egypt

2 Qatar University, Electrical Engineering Department, Doha, Qatar

\section{References}

[1] Eldery, M. A, Saadany, E. F, \& Salama, M. M. A Novel Operator Algorithm for Voltage Envelope Tracking" IEEE Transactions on Power Systems, (2005). , 20(1), 510-512.

[2] Liu, Y, Steurer, M, \& Riberio, P. A Novel Approach to Power Quality Assessment: Real Time hardware-in-the-Loop Test Bed", IEEE Transactions on Power Delivery, (2005). , 20(2), 1200-1201.

[3] Wang, J, Chen, S, \& Lie, T. T. System Voltage Sag Performance Estimation”, IEEE Transactions on Power Delivery (2005). , 20(2), 1738-1747.

[4] Chilukuri, M. V, \& Dash, P. K. Multi-resolution S-Transform-Based Fuzzy Recognition System for Power Quality Events", IEEE Transactions on Power Delivery (2004). , 19(1), 323-330.

[5] Abdel-galil, T. K, Saadany, E. F, \& Salama, M. M. Online Tracking of Voltage Flicker Utilizing Energy Operator and Hilbert Transform" IEEE Transactions on Power Delivery (2004). , 19(2), 861-867.

[6] Wang, F, \& Bollen, M. H. J. Frequency-Response Characteristics and Error Estimation in RMS Measurement", IEEE Transactions on Power Delivery, (2004). , 19(4), 1569-1578.

[7] Marie, M. I, Saadany, E. F, \& Salama, M. M. Envelope Tracking Techniques for Flicker Mitigation and Voltage Regulation", IEEE Transactions on Power Delivery, (2004). , 19(4), 1854-1861.

[8] Liao, Y, Lee, J. B, \& Fuzzy- Expert, A. System for Classifying Power Quality Disturbances" Electrical Power and Energy System, (2004). , 26, 199-205.

[9] Ibrahim, W. R, \& Morcos, M. M. A Power Quality Perspective to System Operational Diagnosis Using Fuzzy Logic and Adaptive Techniques" IEEE Transactions on Power Delivery, (2003). , 18(3), 903-909.

[10] Kezunovic, M, \& Liao, Y. A Novel Software Implementation Concept for Power Quality Study", IEEE Transactions on Power Delivery, (2002). , 17(2), 544-549. 
[11] Anis, W. R. Ibrahim, and M. M. Morcos, “An Adaptive Fuzzy Technique for Learning Power- Quality Signature Waveforms", IEEE Power Engineering Review, January (2001). , 56-58.

[12] Poisson, O, Rioual, P, \& Meunier, M. New Signal Processing Tools Applied to Power Quality Analysis" IEEE Transactions on Power Delivery, (1999). , 14(2), 561-566.

[13] Gaouda, M, Salama, M. M, Sultan, M. R, \& Chikhani, A. Y. Power Quality Detection and Classification Using Wavelet- Multi-resolution Signal Decomposition" IEEE Transactions on Power Delivery, (1999). , 14(4), 1469-1476.

[14] Girgis, A. A, Chang, W. B, \& Makram, E. B. A Digital Recursive Measurement Scheme for on-line Tracking of Power System Harmonics", IEEE Transaction on Power Delivery, (1991). , 6(3), 1153-1160.

[15] Soliman, S. A, Christensen, G. S, \& Naggar, K. M. A State Estimation Algorithm for Identification and Measurement of Power System Harmonics" Electric Power System Research Jr. (1990). , 19, 195-206.

[16] Soliman, A. M. A. L-K. a. n. d. a. r. i, S. A. and K.EL-Naggar, "Digital Dynamic Identification Of Power System Sub-Harmonics Based On The Least Absolute Value," Electric Power Systems Research, Jr. (1993). , 28, 99-104.

[17] Abu, E. A, \& Al-feilat, I. El-Amin, and M. Bettayeb," Power System Harmonic Estimation: A Comparative Study", Electric Power System Research Jr. (1994). , 29, 91-97.

[18] Soliman, S. A, \& Hawary, M. E. Measurement of Power Systems Voltage and Flicker Level for Power Quality Analysis", Int. Jr of Electrical Power \& and Energy Systems, (2000). , 22, 447-450.

[19] Soliman, S. A, Alammari, R. A, \& Hawary, M. E. Frequency and Harmonics Evaluation in Power Networks Using Fuzzy Regression Technique," Electric Power Systems Research Jr., (2003). , 66, 171-177.

[20] Soliman, S. A, Alammari, R. A, Mantawy, A. H, \& Hawary, M. E. On the Application of Park's Transformation for Power System Harmonics Identification and Measurements" Electric Power Systems Components Journal (Formally Electric Machines and Power Systems Journal), (2003). , 31(8)

[21] Soliman, S. A, Mantawy, A. H, \& Hawary, M. E. Simulated Annealing Algorithm for Power System Quality Analysis", International Journal of Electrical Power and Energy Systems, (2004). , 26, 31-36.

[22] Al-kandari, A. M, Soliman, S. A, \& Alammari, R. A. Power Quality Analysis Based on Fuzzy Estimation Algorithm: Voltage Flicker Measurements" Int. Jr. of Electrical Power and Energy Systems, (2006). , 28, 723.

[23] Al-kandari, A. M, Soliman, S. A, \& Measurement, O. F. A POWER SYSTEM NOMINAL VOLTAGE, FREQUENCY AND VOLTAGE FLICKER PARAMETERS" Electri- 
cal Power and Energy Systems (2009). http://www.sciencedirect.com/science/ article/pii/S0142061509000143, 31(2009)

[24] Osowski, S. Neural Network for Estimation of Harmonic Components in a Power System," IEE Proc. C, Gener. Trans. Distrib. (1992). , 139(2), 129-135.

[25] Osowski, S. SVD Technique for Estimating of Harmonic Components in a Power System: A Statistical Approach", IEE Proc. Gener. Trans. Distrib. Vol.5, (1994). , 141, 473-479.

[26] Dash, P. K, Swain, D. P, Liew, A. C, \& Rahman, S. An Adaptive Linear Combiner for on-line Tracking of Power System Harmonics", IEEE Transactions on Power Systems, (1996). , 11(4), 1730-1735.

[27] Smith, B. C, Watson, N. R, Wood, A. R, \& Arrillaga, J. A Newton Solution for the Harmonic Phasor Analysis of AC/DC “, IEEE Trans. on Power Delivery, April (1996). , 11(2), 965-971.

[28] Moreno, V. M. Saiz, J. Barros Guadalupe, “Application of Kalman Filtering for Continuous Real-Time Tracking of Power System Harmonics", IEE Proced on Generation, Transmission and Distribution, Part C Jan.(1997). , 144(1), 13-20.

[29] “Time- Varying Harmonics: Part I- Characterizing Measured Data"Probabilistic Aspect Task Force of the Harmonics Working Group, IEEE Trans on Power Delivery, (1998). , 13(3), 938-944.

[30] Soliman, S. A, Helal, I, \& Al-kandari, A. M. Fuzzy Linear Regression for Measurement of Harmonic Components in a Power System", Electric Power Systems Research (1999). , 50, 99-105.

[31] Bucci, G, \& Landi, C. On-Line Digital Measurement for the Quality Analysis of Power Systems Under Non-sinusoidal Conditions" IEEE Trans. on Instrumentation and Measurement, N0. 4, (1999). , 48, 853-857.

[32] Exposito, A. G. J. A. Rosendo Macias, “ Fast Harmonic Computation for Digital Relaying" IEEE Transaction on Power Delivery, (1999). , 14(4), 1263-1268.

[33] Kim, F, Enbouch, L, Chaouiand, A, \& Michel, M. Novel Identification-Based Technique for Harmonics Estimation", Can. J.Elect. \& Comp.Eng. (1999). , 24(4), 149-154.

[34] Lai, L. L, Chan, W. L, \& Tse, C. T. Real-Time Frequency and Harmonic Evaluation Using Artificial Neural Networks", IEEE Transaction on Power Delivery, (1999). , 14(1), 52-59.

[35] Andreon, S, Yaz, E. E, \& Olejniczak, K. J. Reduced-Order Estimation of Power System Harmonics Using Set Theory", Proceeding of the 1999 IEEE International Conference on Control Application, Hawai'I, USA, August (1999). , 22-27.

[36] Tsao, T. P, Wu, R. C, \& Nig, C. C. The Optimization of Spectral Analysis for Signal Harmonics", IEEE Transaction on Power Delivery, April (2001). , 16(2), 149-153. 
[37] Lin, H. C, \& Lee, C. S. Enhanced FFT-based Parametric Algorithm for Simultaneous Multiple Harmonics Analysis", IEEE Proc.- Gener. Transm. Distrib., May (2001). , 148(3), 209-214.

[38] Liu, Y. Z, \& Chen, S. A Wavelet Based Model for On-Line Tracking of Power System Harmonic Using Kalman Filtering", IEEE Summer Power Meeting, Vancouver, B. C. July (2001). , 17-19.

[39] Skvarenina, T, \& Dewitt, W. Electrical Power and Control", Prentice Hall. Ins., New Jersey, U. S. A, (2001).

[40] Bollen, M. H. J. Understanding Power Quality", IEEE Press Series on Power Engineering, Piscataway, NJ. (2000).

[41] Bollen, M. H. J. Understanding Power Quality", IEEE Press Series on Power Engineering, Piscataway, NJ. (2000).

[42] Oscar, C. Montero-Hernández, and Prasad N. Enjeti, "A Fast Detection Algorithm Suitable for Mitigation of Numerous Power Quality Disturbances', IEEE TRANSACTIONS ON INDUSTRY APPLICATIONS, NOVEMBER/DECEMBER (2005). , 41(6)

[43] Sherif Omar FariedRoy Billinton, and Saleh Aboreshaid," Stochastic Evaluation of Voltage Sag and Unbalance in Transmission Systems" IEEE TRANSACTIONS ON POWER DELIVERY, OCTOBER (2005). , 20(4)

[44] Dong-Jun WonSeon-Ju Ahn, and Seung-Il Moon, "A Modified Sag Characterization Using Voltage Tolerance Curve for Power Quality Diagnosis", IEEE TRANSACTIONS ON POWER DELIVERY, OCTOBER (2005). , 20(4)

[45] Bin WangWilsun Xu, and Zhencun Pan,"Voltage Sag State Estimation for Power Distribution Systems"' IEEE TRANSACTIONS ON POWER SYSTEMS, MAY (2005). , 20(2)

[46] Nicolás Ruiz-ReyesPedro Vera-Candeas, and Francisco Jurado, "Discrimination between Transient Voltage Stability and Voltage Sag Using Damped Sinusoids-Based Transient Modeling IEEE TRANSACTIONS ON POWER DELIVERY, OCTOBER (2005). , 20(4)

[47] Liu, Y, Steurer, M, \& Ribeiro, P. A Novel Approach to Power Quality Assessment: Real Time Hardware-in-the-Loop Test Bed" IEEE TRANSACTIONS ON POWER DELIVERY, APRIL (2005). , 20(2)

[48] Mostafa, I. Marei, Tarek K. Abdel-Galil, Ehab F. El-Saadany, and Magdy M. A. Salama,"Hilbert Transform Based Control Algorithm of the DG Interface for Voltage Flicker Mitigation" IEEE TRANSACTIONS ON POWER DELIVERY, APRIL (2005). , 20(2) 
[49] Eldery, M. A, Saadany, E. F, \& Salama, M. M. A. A Novel Energy Operator Algorithm for Voltage Envelope Tracking", IEEE TRANSACTIONS ON POWER SYSTEMS, FEBRUARY (2005). , 20(1)

[50] Zwe-Lee GaingWavelet-Based Neural Network for Power Disturbance Recognition and Classification", IEEE TRANSACTIONS ON POWER DELIVERY, OCTOBER (2004). , 19(4)

[51] Zhu, T. X, Tso, S. K, \& Lo, K. L. Wavelet-Based Fuzzy Reasoning Approach to PowerQuality Disturbance Recognition" IEEE TRANSACTIONS ON POWER DELIVERY, OCTOBER (2004). , 19(4)

[52] Juan, A. Martinez, and Jacinto Martin-Arnedo, "Voltage Sag Stochastic Prediction Using an Electromagnetic Transients Program," IEEE TRANSACTIONS ON POWER DELIVERY, OCTOBER (2004). , 19(4)

[53] Mostafa, I. Marei, Ehab F. El-Saadany, and Magdy M. A. Salama, "Envelope Tracking Techniques for Flicker Mitigation and Voltage Regulation", EEE TRANSACTIONS ON POWER DELIVERY, OCTOBER (2004). , 19(4)

[54] Chris FitzerMike Barnes, and Peter Green,"Voltage Sag Detection Technique for a Dynamic Voltage Restorer", IEEE TRANSACTIONS ON INDUSTRY APPLICATIONS, JANUARY/FEBRUARY (2004). , 40(1)

[55] Tom, A. Short, Arshad Mansoor, Wes Sunderman, and Ashok Sundaram, "Site Variation and Prediction of Power Quality" IEEE TRANSACTIONS ON POWER DELIVERY, OCTOBER (2003). , 18(4)

[56] Mihaela Albu and GT. Heydt, "On the Use of RMS Values in Power Quality Assessment" IEEE

[57] TRANSACTIONS ON POWER DELIVERYOCTOBER (2003). , 18(4)

[58] Emmanouil StyvaktakisMath H. J. Bollen, and Irene Y. H. Gu, “Expert System for Classification and Analysis of Power System Events" IEEE TRANSACTIONS ON POWER DELIVERY, APRIL (2002). , 17(2)

[59] Olivier PoissonPascal Rioual, and Michel Meunier, 'Detection and Measurement of Power Quality Disturbances Using Wavelet Transform” IEEE TRANSACTIONS ON POWER DELIVERY, JULY (2000). , 15(3)

[60] Anthony, C, \& Parsons, W. Mack Grady, Edward J. Powers, and John C. Soward, "A Direction Finder for Power Quality Disturbances Based Upon Disturbance Power and Energy" IEEE TRANSACTIONS ON POWER DELIVERY, JULY (2000). , 15(3) 